\title{
Exact Solutions of Heat and Mass Transfer with MHD Flow in a Porous Medium under Time Dependent Shear Stress and Temperature
}

\author{
Arshad Khan, ${ }^{1}$ Ilyas Khan, ${ }^{2}$ Farhad Ali, ${ }^{1,3}$ Asma Khalid, ${ }^{1,4}$ and Sharidan Shafie ${ }^{1}$ \\ ${ }^{1}$ Department of Mathematical Sciences, Faculty of Science, Universiti Teknologi Malaysia (UTM), 81310 Johor Bahru, Johor, Malaysia \\ ${ }^{2}$ College of Engineering, Majmaah University, Majmaah 11952, Saudi Arabia \\ ${ }^{3}$ Department of Mathematics, City University of Science and Information Technology, Peshawar 25000, Pakistan \\ ${ }^{4}$ Department of Mathematics, SBK Women's University, Quetta 87300, Pakistan
}

Correspondence should be addressed to Sharidan Shafie; sharidan@utm.my

Received 6 May 2014; Revised 17 July 2014; Accepted 25 July 2014

Academic Editor: Saeed Islam

Copyright (C) 2015 Arshad Khan et al. This is an open access article distributed under the Creative Commons Attribution License, which permits unrestricted use, distribution, and reproduction in any medium, provided the original work is properly cited.

\begin{abstract}
This paper aims to study the influence of thermal radiation on unsteady magnetohyrdodynamic (MHD) natural convection flow of an optically thick fluid over a vertical plate embedded in a porous medium with arbitrary shear stress. Combined phenomenon of heat and mass transfer is considered. Closed-form solutions in general form are obtained by using the Laplace transform technique. They are expressed in terms of exponential and complementary error functions. Velocity is expressed as a sum of thermal and mechanical parts. Corresponding limiting solutions are also reduced from the general solutions. It is found that the obtained solutions satisfy all imposed initial and boundary conditions and reduce to some known solutions from the literature as special cases. Analytical results for the pertinent flow parameters are drawn graphically and discussed in detail. It is found that the velocity profiles of fluid decrease with increasing shear stress. The magnetic parameter develops shear resistance which reduces the fluid motion whereas the inverse permeability parameter increases the fluid flow.
\end{abstract}

\section{Introduction}

Heat and mass transfer process is observed in lots of practical situations, for example, evaporation and chemical reactions as well as condensation. The industrial applications include many transport processes where the simultaneous heat and mass transfer occurs as a result of combined buoyancy effects of thermal diffusion and diffusion of chemical species. Possibly, this is because of the fact that, in many numbers of technical transfer processes, the study of mixed heat and mass transfer is helpful. Few attempts in this direction are made by Singh [1], Narahari [2], Narahari and Nayan [3], Narahari and Ishak [4], Chaudhary and Jain [5], Das et al. [6], Soundalgekar et al. [7], and Muthucumaraswamy et al. [8,9]. A few late efforts in the same area of research are presented in [4-9]. Significant concern has been originated in the study of magnetic field and the electrically conducting fluids flow, while medium is porous [10]. The unsteady free convection fluid flows which are incompressible and viscous near a porous infinite plate with arbitrary time dependent heating plate are investigated by Toki and Tokis [11]. The results of chemical reaction of viscous fluid which are electrically conducting through a porous medium in two-dimensional steady free convection flow past a vertical surface with slip flow region have been presented by Senapati et al. [12]. MHD free convection flow of an incompressible viscous fluid near an oscillating plate embedded in a porous medium has been presented by Khan et al. [13]. Therefore, many researchers have studied free convection flow past a vertical plate with thermal radiation [14-16].

Moreover, several research papers on free convection fluid flows with different thermal conditions at the bounding plate which are continuous and well-defined at the wall are found. However, a number of problems seem with different conditions at the wall. Therefore, its investigation under step change in wall temperature is meaningful. The physical significance of this thought can be seen in the fabrication of 


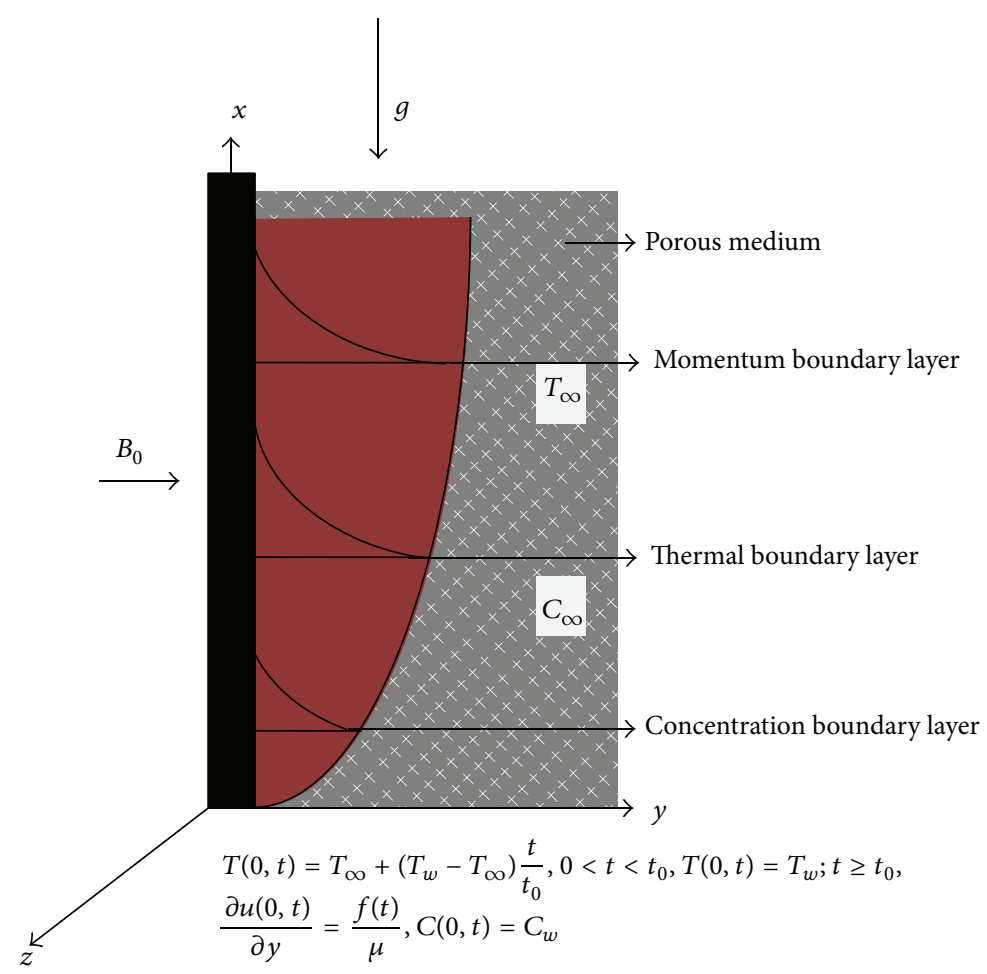

FIgURE 1: Physical configuration of the problem.

thin-film photovoltaic devices [17]. Whenever the conventional supposition of periodic outdoor conditions may lead to substantial errors in the case of a significant temporary deviation of the temperature from periodicity, such as in air conditioning, periodic step changes in temperature are important [18]. Here some of recent and important contributions [18-24] are presented.

Fluid flow past an infinite plate is of much importance due to its large practical applications. Such motion is due to many effects such as motions due to wall shear stress. Closedform results of the problems with shear stress on the wall are difficult; therefore a very rare research is found in the literature. Slip velocity depends on the shear stress linearly; this idea was presented by Navier [25]. Free convection flow near a vertical plate that applies arbitrary shear stress to the fluid was investigated by Fetecau et al. [26]. However, as yet no research has been presented earlier in the literature which mainly focuses on the free convection conjugate flow with thermal diffusion, while taking arbitrary shear stress along ramped wall temperature.

Therefore, exact solutions for MHD conjugate flow of a viscous fluid past a vertical plate that applies arbitrary shear stress to the fluid are presented in this paper. Exact solutions of the initial and boundary value problems that govern the flow are obtained by using Laplace transform technique. From general solutions some of special and limiting cases are derived. The results for velocity field, the temperature field, and concentration field are shown graphically and discussed for different embedded parameters.

\section{Mathematical Formulation}

Consider the unsteady MHD free convection flow of an incompressible viscous fluid over an infinite vertical plate. The geometry of the problem is presented in Figure 1. The plate is along the $x$-axis and the $y$-axis is assumed normal to it. The plate and the fluid are at stationary positions with the constant temperature $T_{\infty}$ and concentration $C_{\infty}$. The fluid experiences shear stress $f(t)$ by the plate after $t=0^{+}$. In the meantime, the plate temperature is aroused or let down to $T_{\infty}+\left(T_{w}-T_{\infty}\right)\left(t / t_{0}\right)$, when $t \leq t_{0}$, and thereafter, for $t>t_{0}$, is kept at constant temperature $T_{w}$ and concentration is aroused to $C_{w}$. The radiation is taken in the energy equation. However, the radiative heat flux is assumed negligible in $x$-direction. We suppose that the fluid flow is laminar, grey absorbingemitting radiation but the medium is with no scattering. Furthermore, we suppose that the fluid is electrically conducting. Hence, we take the following Maxwell equations:

$$
\begin{aligned}
\operatorname{div} \mathbf{B} & =\mathbf{0}, \\
\operatorname{Curl} \mathbf{E} & =-\frac{\partial \mathbf{B}}{\partial t}, \\
\operatorname{Curl} \mathbf{B} & =\mu_{e} \mathbf{J} .
\end{aligned}
$$

In the above equations, $\mathbf{B}, \mathbf{E}$, and $\mu_{e}$ are the magnetic field, electric field intensity, and the magnetic permeability of the fluid, respectively. By using Ohm's law, the current density J is given as

$$
\mathbf{J}=\sigma(\mathbf{E}+\mathbf{V} \times \mathbf{B})
$$


where $\sigma$ is the electrical conductivity of the fluid. Further we make the following assumptions.

(i) $\sigma, \rho, \mu_{e}$ are constants throughout the flow field.

(ii) $\mathbf{B}$ is perpendicular to $\mathbf{V}$.

(iii) The induced magnetic field $\mathbf{b}$ is negligible compared with the imposed magnetic field $\mathbf{B}_{0}$.

(iv) The magnetic Reynolds number is small.

(v) The electric field is zero.

Therefore, the linearized form of the electromagnetic body force [27] is

$$
\frac{1}{\rho} \mathbf{J} \times \mathbf{B}=\frac{\sigma}{\rho}\left[\left(\mathbf{V} \times \mathbf{B}_{0}\right) \times \mathbf{B}_{0}\right]=-\frac{\sigma B_{0}^{2} \mathbf{V}}{\rho} .
$$

Using Boussinesq's approximation and neglecting the viscous dissipation, the equations governing the flow are given by [2]

$$
\begin{aligned}
\frac{\partial u}{\partial t}= & \nu \frac{\partial^{2} u}{\partial y^{2}}+g \beta_{T}\left(T-T_{\infty}\right)+g \beta_{C}\left(C-C_{\infty}\right) \\
& -\frac{\nu}{K} u-\frac{\sigma B_{0}^{2}}{\rho} u ; \quad y, t>0, \\
\rho C_{p} \frac{\partial T}{\partial t}= & k \frac{\partial^{2} T}{\partial y^{2}}-\frac{\partial q_{r}}{\partial y} \quad y, t>0 \\
\frac{\partial C}{\partial t}= & D \frac{\partial^{2} C}{\partial y^{2}}+D_{1} \frac{\partial^{2} T}{\partial y^{2}} \quad y, t>0
\end{aligned}
$$

where $u, C, T, v, g, \rho, \beta_{C}, \beta_{T}, \sigma, C_{p}, B_{0}, k, q_{r}, D$, $D_{1}$, and $K$ are the fluid velocity in $x$-direction, the fluid concentration, the fluid temperature, its kinematic viscosity, the gravitational acceleration, the constant density, the mass transfer coefficient, the heat transfer coefficient, the fluid electric conductivity, the heat capacity, the applied magnetic field, the thermal conductivity, the radiative heat flux, mass diffusivity, thermal diffusivity, and the permeability of the porous medium.

The corresponding initial and boundary conditions are

$$
\begin{aligned}
& u(y, 0)=0, \\
& T(y, 0)=T_{\infty}, \\
& C(y, 0)=C_{\infty} ; \\
& \forall y \geqslant 0, \\
& \frac{\partial u(0, t)}{\partial y}=\frac{f(t)}{\mu}, \\
& C(0, t)=C_{w} ; \\
& t>0,
\end{aligned}
$$

$$
\begin{aligned}
T(0, t) & =T_{\infty}+\left(T_{w}-T_{\infty}\right) \frac{t}{t_{0}}, \quad 0<t<t_{0} \\
T(0, t) & =T_{w} ; \quad t \geq t_{0} \\
u(\infty, t) & =0 \\
T(\infty, t) & =T_{\infty} \\
C(\infty, t) & =C_{\infty} ;
\end{aligned}
$$

$t>0$.

The radiation heat flux under Rosseland approximation for optically thick fluid [28] is given by

$$
q_{r}=-\frac{4 \sigma^{*}}{3 k_{R}} \frac{\partial T^{4}}{\partial y}
$$

where $\sigma^{*}$ and $k_{R}$ are the Stefan-Boltzman constant and the mean absorption coefficient. We can see from (8) that the radiation term is nonlinear. Recently David Maxim Gururaj and Anjali Devi [29] used nonlinear radiation effects and studied MHD boundary layer flow with forced convection past a nonlinearly stretching surface with variable temperature. Therefore, we follow David Maxim Gururaj and Anjali Devi [29] and assume that the temperature differences within the flow are sufficiently small; that is, the difference between the fluid temperature and the free stream temperature is negligible, so that (8) can be linearized by expanding $T$ into the Taylor series about $T_{\infty}$, which after neglecting higher order terms takes the form

$$
T^{4} \approx 4 T_{\infty}^{3} T-3 T_{\infty}^{4} .
$$

Introducing (5), (8), and (9), we get

$$
\operatorname{Pr} \frac{\partial T}{\partial t}=\nu\left(1+N_{r}\right) \frac{\partial^{2} T}{\partial y^{2}} ; \quad y, t>0,
$$

where $\operatorname{Pr}, \nu$, and $N_{r}$ are defined by

$$
\begin{aligned}
\operatorname{Pr} & =\frac{\mu C_{p}}{k}, \\
\nu & =\frac{\mu}{\rho}, \\
N_{r} & =\frac{16 \sigma T_{\infty}^{3}}{3 k k_{R}} .
\end{aligned}
$$

Taking the nondimensional variables

$$
\begin{aligned}
u^{*} & =u \sqrt{\frac{t_{0}}{v}}, \\
T^{*} & =\frac{T-T_{\infty}}{T_{w}-T_{\infty}}, \\
C^{*} & =\frac{C-C_{\infty}}{C_{w}-C_{\infty}},
\end{aligned}
$$




$$
\begin{aligned}
y^{*} & =\frac{y}{\sqrt{v t_{0}},} \\
t^{*} & =\frac{t}{t_{0}}, \\
f^{*}\left(t^{*}\right) & =\frac{t_{0}}{\mu} f(t),
\end{aligned}
$$

by eliminating the star notations into (4), (6), and (10), we obtain

$$
\begin{aligned}
\frac{\partial u}{\partial t} & =\frac{\partial^{2} u}{\partial y^{2}}+\mathrm{Gr} T+\mathrm{GmC}-K_{p} u-M u, \\
\operatorname{Pr}_{\mathrm{eff}} \frac{\partial T}{\partial t} & =\frac{\partial^{2} T}{\partial y^{2}}, \\
\frac{\partial C}{\partial t} & =\frac{1}{\mathrm{Sc}} \frac{\partial^{2} C}{\partial y^{2}}+\operatorname{Sr} \frac{\partial^{2} T}{\partial y^{2}},
\end{aligned}
$$

where $\operatorname{Pr}_{\text {eff }}=\operatorname{Pr} /\left(1+N_{r}\right)$ is the effective Prandtl number [28, Equation (10)] and

$$
\begin{aligned}
\mathrm{Gr} & =\frac{g \beta_{T}\left(T_{w}-T_{\infty}\right) v}{U_{0}^{3}}, \\
\mathrm{Gm} & =\frac{g \beta_{c}\left(C_{w}-C_{\infty}\right) v}{U_{0}^{3}}, \\
M & =\frac{\sigma B_{0}^{2} t_{0}}{\rho}, \\
\mathrm{Sc} & =\frac{v}{D}, \\
K_{p} & =\frac{v t_{0}}{K}, \\
t_{0} & =\frac{v}{U_{0}^{2}}, \\
\mathrm{Sr} & =\frac{D_{1}\left(T_{w}-T_{\infty}\right)}{\left(C_{w}-C_{\infty}\right) v}
\end{aligned}
$$

are the Grashof number, modified Grashof number, magnetic parameter, Schmidt number, the inverse permeability parameter for the porous medium, the characteristic time, and Soret number, respectively.

The nondimensional initial and boundary conditions are

$$
\begin{gathered}
u(y, 0)=0, \\
T(y, 0)=0, \\
C(y, 0)=0 ; \\
\forall y \geq 0, \\
\left.\frac{\partial u}{\partial y}\right|_{y=0}=f(t),
\end{gathered}
$$

$$
\begin{aligned}
T(0, t) & =t \\
0 & <t \leq 1, \\
T(0, t) & =1 ; \quad t>1, \\
C(0, t) & =1 \\
C(\infty, t) & =0 \\
T(\infty, t) & =0 \\
u(\infty, t) & =0
\end{aligned}
$$$$
t>0 \text {. }
$$

\section{Solution of the Problem}

To solve (13) under conditions (15), by taking Laplace transform technique, we obtained

$$
\begin{aligned}
q \bar{u}(y, q)= & \frac{\partial^{2} \bar{u}(y, q)}{\partial y^{2}}+\operatorname{Gr} \bar{T}(y, q)+\operatorname{Gm} \bar{C}(y, q) \\
& -K_{p} \bar{u}(y, q)-M \bar{u}(y, q) \\
\bar{T}(y, q)= & \frac{1}{\operatorname{Pr}_{\text {eff }} q} \frac{\partial^{2} \bar{T}(y, q)}{\partial y^{2}} \\
\bar{C}(y, q)= & \frac{1}{\operatorname{Sc} q} \frac{\partial^{2} \bar{C}(y, q)}{\partial y^{2}}+\frac{\operatorname{Sr}}{q} \frac{\partial^{2} \bar{T}(y, q)}{\partial y^{2}}
\end{aligned}
$$

with boundary conditions

$$
\begin{aligned}
\bar{C}(\infty, q) & =0, \\
\bar{C}(0, q) & =\frac{1}{q}, \\
\bar{T}(\infty, q) & =0, \\
\bar{u}(\infty, q) & =0, \\
\left.\frac{\partial \bar{u}(y, q)}{\partial y}\right|_{y=0} & =F(q), \\
T(0, q) & =\frac{1-e^{-q}}{q^{2}} .
\end{aligned}
$$

Solving (17) using (19), we get

$$
\bar{T}(y, q)=\frac{1}{q^{2}} e^{-y \sqrt{q \mathrm{Pr}_{\mathrm{eff}}}}-\frac{e^{-q}}{q^{2}} e^{-y \sqrt{q \mathrm{Pr}_{\mathrm{eff}}}},
$$

by inverse Laplace transform giving

$$
T(y, t)=f(y, t)-f(y, t-1) H(t-1),
$$


where

$$
\begin{aligned}
f(y, t)= & \left(\frac{\operatorname{Pr}_{\text {eff }} y^{2}}{2}+t\right) \operatorname{erf} c\left(\frac{\sqrt{\operatorname{Pr}_{\text {eff }}} y}{2 \sqrt{t}}\right) \\
& -\sqrt{\frac{\operatorname{Pr}_{\text {eff }} t}{\pi}} y \exp \left(\frac{-\operatorname{Pr}_{\text {eff }} y^{2}}{4 t}\right), \\
\left.\frac{\partial T(y, t)}{\partial y}\right|_{y=0}= & \frac{2 \sqrt{\operatorname{Pr}_{\text {eff }}}}{\sqrt{\pi}}(\sqrt{t}-\sqrt{t-1} H(t-1)),
\end{aligned}
$$

which is the Nusselt number. Error complementary error functions of Gauss [30] are denoted by $\operatorname{erf}(\cdot)$ and $\operatorname{erfc}(\cdot)$.

Solution of (18) under boundary conditions (19) yields

$$
\begin{aligned}
C(y, q)= & \frac{1}{q} e^{-y \sqrt{\mathrm{Scq}}}+\frac{c_{18}\left(1-e^{-q}\right)}{q^{2}} e^{-y \sqrt{\mathrm{Sc}} \sqrt{q}} \\
& -\frac{c_{18}\left(1-e^{-q}\right)}{q^{2}} e^{-y \sqrt{\mathrm{P}_{\mathrm{eff}}} \sqrt{q}}
\end{aligned}
$$

by taking inverse Laplace transform giving

$$
\begin{aligned}
& C(y, t)=\operatorname{erf} c\left(\frac{y \sqrt{\mathrm{Sc}}}{2 \sqrt{t}}\right)+c_{18}\left(\left(t+\frac{\mathrm{Sc} y^{2}}{2}\right) \operatorname{erf} c\right. \\
& \left.\cdot\left(\frac{y \sqrt{S c}}{2 \sqrt{t}}\right)-\frac{y \sqrt{S c} \sqrt{t}}{\sqrt{\pi}} e^{-y^{2} S c / 4 t}\right)-c_{18}((t \\
& \left.+\frac{\operatorname{Pr}_{\text {eff }} y^{2}}{2}\right) \operatorname{erfc}\left(\frac{y \sqrt{\operatorname{Pr}_{\text {eff }}}}{2 \sqrt{t}}\right)-\frac{y \sqrt{\operatorname{Pr}_{\text {eff }}} \sqrt{t}}{\sqrt{\pi}} \\
& \left.\cdot e^{-y^{2} \mathrm{Pr}_{\mathrm{eff}} / 4 t}\right) \\
& +\left[c _ { 1 8 } \left(\left(t-1+\frac{\operatorname{Pr}_{\text {eff }} y^{2}}{2}\right) \operatorname{erfc}\left(\frac{y \sqrt{\operatorname{Pr}_{\text {eff }}}}{2 \sqrt{t-1}}\right)\right.\right. \\
& \left.\left.-\frac{y \sqrt{\operatorname{Pr}_{\text {eff }}} \sqrt{t-1}}{\sqrt{\pi}} e^{-y^{2} \operatorname{Pr}_{\text {eff }} / 4(t-1)}\right)\right] H(t-1) \\
& -\left[c _ { 1 8 } \left(\left(t-1+\frac{\mathrm{Sc} y^{2}}{2}\right) \operatorname{erfc}\left(\frac{y \sqrt{\mathrm{Sc}}}{2 \sqrt{t-1}}\right)\right.\right. \\
& \left.\left.-\frac{y \sqrt{\mathrm{Sc}} \sqrt{t-1}}{\sqrt{\pi}} e^{-y^{2} \mathrm{Sc} / 4(t-1)}\right)\right] H(t-1), \\
& \left.\frac{\partial C(y, t)}{\partial y}\right|_{y=0}=\frac{-2 \sqrt{\mathrm{Sc}}-3 c_{18} \sqrt{\mathrm{Sc} t}}{\sqrt{\pi t}} \\
& -\left[\frac{-2 \sqrt{\mathrm{Sc}}-3 c_{18} \sqrt{\mathrm{Sc}}(t-1)}{\sqrt{\pi(t-1)}}\right] H(t-1),
\end{aligned}
$$

which is the corresponding mass transfer rate also known as Sherwood number.
The solution of (16) under boundary conditions (19) results in

$$
\begin{aligned}
\bar{u}(y, q)= & \frac{c_{2} \sqrt{q}\left(1-e^{-q}\right)}{q^{2}\left(q-c_{1}\right) \sqrt{q+H_{1}}} e^{-y \sqrt{q+H_{1}}} \\
& +\frac{c_{4} \sqrt{q}}{q\left(q-c_{3}\right) \sqrt{q+H_{1}}} e^{-y \sqrt{q+H_{1}}} \\
& -\frac{F(q)}{\sqrt{q+H_{1}}} e^{-y \sqrt{q+H_{1}}} \\
& +\frac{c_{6} \sqrt{q}\left(1-e^{-q}\right)}{q^{2}\left(q-c_{5}\right) \sqrt{q+H_{1}}} e^{-y \sqrt{q+H_{1}}} \\
& -\frac{c_{7} \sqrt{q}\left(1-e^{-q}\right)}{q^{2}\left(q-c_{1}\right) \sqrt{q+H_{1}}} e^{-y \sqrt{q+H_{1}}} \\
& -\frac{c_{8}\left(1-e^{-q}\right)}{q^{2}\left(q-c_{1}\right)} e^{-y \sqrt{q \mathrm{P}_{\mathrm{eff}}}} \\
& +\frac{c_{11}\left(1-e^{-q}\right)}{q^{2}\left(q-c_{1}\right)} e^{-y \sqrt{q \mathrm{P}_{\mathrm{eff}}}} \\
& -\frac{c_{10}\left(1-e^{-q}\right)}{q^{2}\left(q-c_{5}\right)} e^{-y \sqrt{q S \mathrm{sc}}}-\frac{c_{9}}{q\left(q-c_{3}\right)} e^{-y \sqrt{q S c}}
\end{aligned}
$$

which upon inverse Laplace transform results in

$$
u(y, t)=u_{c}(y, t)+u_{m}(y, t),
$$

where $u_{c}(y, t)$ corresponds to convective part of velocity which is defined as

$$
\begin{aligned}
& u_{c}(y, t)=V_{1}+V_{2}, \\
& V_{1}=c_{2} \int_{0}^{t}\left(\frac{e^{c_{1}(t-s)} \operatorname{erf}\left(\sqrt{c_{1}(t-s)}\right)}{\left(c_{1}\right)^{3 / 2}}-\frac{2 \sqrt{t-s}}{\sqrt{\pi} c_{1}}\right) \\
& \cdot \frac{e^{-H_{1} s-y^{2} / 4 s}}{\sqrt{\pi s}} d s-\left[\frac{c_{2}}{\left(c_{1}\right)^{3 / 2} \sqrt{\pi}}\right. \\
& \left.\cdot \int_{0}^{t-1} \frac{e^{c_{1}(t-1-s)-H_{1} s-y^{2} / 4 s} \operatorname{erf}\left(\sqrt{c_{1}(t-1-s)}\right)}{\sqrt{s}} d s\right] \\
& \cdot H(t-1)+\left[\frac{c_{2}}{c_{1} \pi}\right. \\
& \left.\cdot \int_{0}^{t-1} \frac{(2 \sqrt{t-1-s}) e^{-H_{1} s-y^{2} / 4 s}}{\sqrt{s}} d s\right] H(t-1)
\end{aligned}
$$




$$
\begin{aligned}
& -c_{7} \int_{0}^{t}\left(\frac{e^{c_{1}(t-s)} \operatorname{erf}\left(\sqrt{c_{1}(t-s)}\right)}{\left(c_{1}\right)^{3 / 2}}-\frac{2 \sqrt{t-s}}{\sqrt{\pi} c_{1}}\right) \\
& \cdot \operatorname{erf} c\left(\frac{y \sqrt{\mathrm{Sc}}}{2 \sqrt{t}}-\sqrt{c_{3} t}\right)-\frac{c_{9} e^{c_{3} t+y \sqrt{c_{3} \mathrm{Sc}}}}{2 c_{3}} \\
& \cdot \frac{e^{-H_{1} s-y^{2} / 4 s}}{\sqrt{\pi s}} d s+\left[\frac{c_{7}}{\left(c_{1}\right)^{3 / 2} \sqrt{\pi}}\right. \\
& \cdot \operatorname{erf} c\left(\frac{y \sqrt{\mathrm{Sc}}}{2 \sqrt{t}}+\sqrt{c_{3} t}\right) \\
& \left.\cdot \int_{0}^{t-1} \frac{e^{c_{1}(t-1-s)-H_{1} s-y^{2} / 4 s} \operatorname{erf}\left(\sqrt{c_{1}(t-1-s)}\right)}{\sqrt{s}} d s\right] \\
& V_{2}=\frac{c_{10}}{c_{3}}\left(t+\frac{\mathrm{Sc} y^{2}}{2}\right) \operatorname{erf} c\left(\frac{y \sqrt{\mathrm{Sc}}}{2 \sqrt{t}}\right)-\left[\frac{c_{10}}{c_{3}^{2}}\right. \\
& \left.\cdot \operatorname{erf} c\left(\frac{y \sqrt{\mathrm{Sc}}}{2 \sqrt{t-1}}\right)\right] H(t-1) \\
& -\frac{\left(c_{8}-c_{11}\right) e^{c_{1} t-y \sqrt{\mathrm{Pr}_{\text {eff }} c_{1}}}}{2 c_{1}^{2}} \operatorname{erf} c\left(\frac{y \sqrt{\mathrm{Pr}_{\text {eff }}}}{2 \sqrt{t}}-\sqrt{c_{1} t}\right) \\
& -\frac{\left(c_{8}-c_{11}\right) e^{c_{1} t+y \sqrt{\mathrm{Pr}_{\mathrm{eff}} c_{1}}}}{2 c_{1}^{2}} \operatorname{erf} c\left(\frac{y \sqrt{\mathrm{Pr}_{\mathrm{eff}}}}{2 \sqrt{t}}+\sqrt{c_{1} t}\right) \\
& +\left[\frac{c_{10}}{c_{3}} \frac{y \sqrt{\mathrm{Sc}} \sqrt{t-1}}{\sqrt{\pi}} e^{-y^{2} \mathrm{Sc} / 4(t-1)}\right] H(t-1)-\frac{c_{10}}{c_{3}} \\
& \cdot \frac{y \sqrt{\mathrm{Sc}} \sqrt{t}}{\sqrt{\pi}} e^{-y^{2} \mathrm{Sc} / 4 t}+\frac{c_{10}}{c_{3}^{2}} \operatorname{erf} c\left(\frac{y \sqrt{\mathrm{Sc}}}{2 \sqrt{t}}\right) \\
& -\frac{\left(c_{8}-c_{11}\right)}{c_{1}} \frac{y \sqrt{\operatorname{Pr}_{\text {eff }}} \sqrt{t}}{\sqrt{\pi}} e^{-y^{2} \mathrm{Pr}_{\text {eff }} / 4 t}+\frac{\left(c_{8}-c_{11}\right)}{c_{1}^{2}} \\
& \cdot \operatorname{erf} c\left(\frac{y \sqrt{\operatorname{Pr}_{\mathrm{eff}}}}{2 \sqrt{t}}\right)-\left[\frac{\left(c_{8}-c_{11}\right)}{c_{1}^{2}} \operatorname{erf} c\left(\frac{y \sqrt{\mathrm{Pr}_{\mathrm{eff}}}}{2 \sqrt{t-1}}\right)\right] \\
& \cdot H(t-1)+\left[\frac{c_{10} e^{c_{3}(t-1)-y \sqrt{\mathrm{Scc}_{3}}}}{2 c_{3}^{2}}\right. \\
& \left.\cdot \int_{0}^{t-1} \frac{(2 \sqrt{t-1-s}) e^{-H_{1} s-y^{2} / 4 s}}{\sqrt{s}} d s\right] H(t-1) \\
& +c_{4} \int_{0}^{t}\left(e^{c_{3}(t-s)} \operatorname{erf}\left(\sqrt{c_{3}(t-s)}\right)\right) \frac{e^{-H_{1} s-y^{2} / 4 s}}{\sqrt{c_{3} \pi s}} d s \\
& +\frac{c_{8}}{c_{1}}\left(t+\frac{\operatorname{Pr}_{\text {eff }} y^{2}}{2}\right) \operatorname{erf} c\left(\frac{y \sqrt{\operatorname{Pr}_{\text {eff }}}}{2 \sqrt{t}}\right) \\
& -\left[\frac{c_{8}}{c_{1}}\left(t-1+\frac{\operatorname{Pr}_{\mathrm{eff}} y^{2}}{2}\right) \operatorname{erf} c\left(\frac{y \sqrt{\mathrm{Pr}_{\mathrm{eff}}}}{2 \sqrt{t-1}}\right)\right] H(t \\
& -1)+\left[\frac{c_{8}}{c_{1}} \frac{y \sqrt{\operatorname{Pr}_{\text {eff }}} \sqrt{t-1}}{\sqrt{\pi}} e^{-y^{2} \operatorname{Pr}_{\text {eff }} / 4(t-1)}\right] H(t-1) \\
& -\frac{c_{9} e^{c_{3} t-y \sqrt{c_{3} \mathrm{Sc}}}}{2 c_{3}} \operatorname{erf} c\left(\frac{y \sqrt{\mathrm{Sc}}}{2 \sqrt{t}}-\sqrt{c_{3} t}\right)+\frac{c_{9}}{c_{3}} \\
& \cdot \operatorname{erf} c\left(\frac{y \sqrt{\mathrm{Sc}}}{2 \sqrt{t}}\right)-\frac{c_{10} e^{c_{3} t-y \sqrt{\mathrm{Scc}_{3}}}}{2 c_{3}^{2}} \\
& \left.\cdot \operatorname{erf} c\left(\frac{y \sqrt{\mathrm{Sc}}}{2 \sqrt{(t-1)}}-\sqrt{c_{3}(t-1)}\right)\right] H(t-1) \\
& -\frac{c_{10} e^{c_{3} t+y \sqrt{S c c_{3}}}}{2 c_{3}^{2}} \operatorname{erf} c\left(\frac{y \sqrt{\mathrm{Sc}}}{2 \sqrt{t}}+\sqrt{c_{3} t}\right) \\
& -\left[\frac{c_{10}}{c_{3}}\left(t-1+\frac{\mathrm{Sc} y^{2}}{2}\right) \operatorname{erf} c\left(\frac{y \sqrt{\mathrm{Sc}}}{2 \sqrt{t-1}}\right)\right] H(t \\
& -1)+\left[\frac{\left(c_{8}-c_{11}\right) e^{c_{1}(t-1)-y \sqrt{\mathrm{Pr}_{\mathrm{eff}} c_{1}}}}{2 c_{1}^{2}}\right. \\
& \left.\cdot \operatorname{erf} c\left(\frac{y \sqrt{\operatorname{Pr}_{\mathrm{eff}}}}{2 \sqrt{(t-1)}}-\sqrt{c_{1}(t-1)}\right)\right] H(t-1) \\
& +\left[\frac{\left(c_{8}-c_{11}\right) e^{c_{1}(t-1)+y \sqrt{\operatorname{Pr}_{\text {eff }} c_{1}}}}{2 c_{1}^{2}}\right. \\
& \left.\cdot \operatorname{erf} c\left(\frac{y \sqrt{\operatorname{Pr}_{\text {eff }}}}{2 \sqrt{(t-1)}}+\sqrt{c_{1}(t-1)}\right)\right] H(t-1)
\end{aligned}
$$


and $u_{m}(y, t)$ is mechanical part of velocity defined as

$$
u_{m}(y, t)=-\frac{1}{\sqrt{\pi}} \int_{0}^{t} \frac{f(t-s) e^{-H_{1} s-y^{2} / 4 s}}{\sqrt{s}}
$$

where

$$
\begin{aligned}
& c_{1}=\frac{H_{1}}{\operatorname{Pr}_{\mathrm{eff}}-1}, \\
& c_{2}=\frac{\mathrm{Gr} \sqrt{\operatorname{Pr}_{\mathrm{eff}}}}{\operatorname{Pr}_{\mathrm{eff}}-1}, \\
& c_{3}=\frac{H_{1}}{\mathrm{Sc}-1}, \\
& c_{4}=\frac{\mathrm{Gm} \sqrt{\mathrm{Sc}}}{\mathrm{Sc}-1}, \\
& c_{6}=\frac{\mathrm{GmScSrPr}_{\mathrm{eff}} \sqrt{\mathrm{Sc}}}{\mathrm{Sc}-1} \text {, } \\
& c_{7}=\frac{\mathrm{GmScSrPr}_{\mathrm{eff}} \sqrt{\mathrm{Pr}_{\mathrm{eff}}}}{\operatorname{Pr}_{\mathrm{eff}}-1} \text {, } \\
& c_{8}=\frac{\mathrm{Gr}}{\operatorname{Pr}_{\mathrm{eff}}-1} \text {, } \\
& c_{9}=\frac{\mathrm{Gm}}{\mathrm{Sc}-1} \text {, } \\
& c_{10}=\frac{\mathrm{GmScSrPr}_{\mathrm{eff}}}{\mathrm{Sc}-1} \text {, } \\
& c_{11}=\frac{\mathrm{GmScSrPr}_{\mathrm{eff}}}{\mathrm{Pr}_{\mathrm{eff}}-1} \text {, } \\
& c_{12}=\frac{M}{\operatorname{Pr}_{\text {eff }}-1}, \\
& c_{13}=\frac{M}{\mathrm{Sc}-1} \text {, } \\
& c_{15}=\frac{K_{p}}{\operatorname{Pr}_{\mathrm{eff}}-1} \text {, } \\
& c_{16}=\frac{K_{p}}{\mathrm{Sc}-1} \text {, } \\
& c_{18}=\mathrm{SrScPr}_{\mathrm{eff}} \text {, } \\
& H_{1}=K_{p}+M \text {, } \\
& c_{19}=\frac{\mathrm{GmSc}^{3 / 2} \mathrm{SrPr}_{\mathrm{eff}}}{\left(\mathrm{Pr}_{\mathrm{eff}}-\mathrm{Sc}\right)(\mathrm{Sc}-1)} \text {, } \\
& c_{20}=\frac{\mathrm{GmPr}_{\mathrm{eff}}^{3 / 2} \mathrm{SrSc}}{\left(\mathrm{Pr}_{\mathrm{eff}}-\mathrm{Sc}\right)(\mathrm{Sc}-1)} \text {, } \\
& c_{21}=\frac{\mathrm{GmPr}_{\mathrm{eff}} \mathrm{SrSc}}{\left(\mathrm{Pr}_{\mathrm{eff}}-\mathrm{Sc}\right)(\mathrm{Sc}-1)} \text {. }
\end{aligned}
$$

\section{Plate with Constant Temperature}

The solution of (17) under boundary conditions (19) for constant temperature yields

$$
\begin{aligned}
T(y, t) & =\operatorname{erf} c\left(\frac{y \sqrt{\mathrm{Pr}_{\mathrm{eff}}}}{2 \sqrt{t}}\right), \\
\frac{\partial T(0, t)}{\partial y} & =-\frac{\sqrt{\mathrm{Pr}_{\mathrm{eff}}}}{\sqrt{\pi t}} .
\end{aligned}
$$

The solution of (16) under boundary conditions (19) for constant temperature is

$$
\begin{aligned}
\bar{u}(y, q)= & \frac{c_{2} \sqrt{q}}{q\left(q-c_{1}\right) \sqrt{q+H_{1}}} e^{-y \sqrt{q+H_{1}}} \\
& +\frac{c_{4} \sqrt{q}}{q\left(q-c_{3}\right) \sqrt{q+H_{1}}} e^{-y \sqrt{q+H_{1}}} \\
& -\frac{F(q)}{\sqrt{q+H_{1}}} e^{-y \sqrt{q+H_{1}}} \\
& +\frac{c_{19} \sqrt{q}}{q\left(q-c_{3}\right) \sqrt{q+H_{1}}} e^{-y \sqrt{q+H_{1}}} \\
& -\frac{c_{8}}{q\left(q-c_{1}\right)} e^{-y \sqrt{q \mathrm{Pr}_{\mathrm{eff}}}} \\
& -\frac{c_{20} \sqrt{q}}{q\left(q-c_{1}\right) \sqrt{q+H_{1}}} e^{-y \sqrt{q+H_{1}}} \\
& -\frac{c_{21}}{q\left(q-c_{3}\right)} e^{-y \sqrt{q S \mathrm{sc}}}+\frac{c_{21}}{q\left(q-c_{1}\right)} e^{-y \sqrt{q \mathrm{Pr}_{\mathrm{eff}}}} \\
& -\frac{c_{9}}{q\left(q-c_{3}\right)} e^{-y \sqrt{q S \mathrm{sc}}},
\end{aligned}
$$

upon inverse Laplace transform

$$
u(y, t)=u_{c}(y, t)+u_{m}(y, t)
$$

where

$$
\begin{aligned}
& u_{c}(y, t)=V_{3}+V_{4}, \\
& V_{3} \\
& =c_{2} \int_{0}^{t}\left(e^{c_{1}(t-s)} \operatorname{erf}\left(\sqrt{c_{1}(t-s)}\right)\right) \frac{e^{-H_{1} s-y^{2} / 4 s}}{\sqrt{c_{1} \pi s}} d s \\
& \quad+c_{4} \int_{0}^{t}\left(e^{c_{3}(t-s)} \operatorname{erf}\left(\sqrt{c_{3}(t-s)}\right)\right) \frac{e^{-H_{1} s-y^{2} / 4 s}}{\sqrt{c_{3} \pi s}} d s
\end{aligned}
$$




$$
\begin{aligned}
& +c_{19} \int_{0}^{t}\left(e^{c_{3}(t-s)} \operatorname{erf}\left(\sqrt{c_{3}(t-s)}\right)\right) \frac{e^{-H_{1} s-y^{2} / 4 s}}{\sqrt{c_{3} \pi s}} d s \\
& -c_{20} \int_{0}^{t}\left(e^{c_{1}(t-s)} \operatorname{erf}\left(\sqrt{c_{1}(t-s)}\right)\right) \frac{e^{-H_{1} s-y^{2} / 4 s}}{\sqrt{c_{1} \pi s}} d s \\
& -\frac{c_{21} e^{c_{3} t-y \sqrt{c_{3} S c}}}{2 c_{3}} \operatorname{erf} c\left(\frac{y \sqrt{\mathrm{Sc}}}{2 \sqrt{t}}-\sqrt{c_{3} t}\right) \\
& +\frac{c_{21}}{c_{3}} \operatorname{erf} c\left(\frac{y \sqrt{\mathrm{Sc}}}{2 \sqrt{t}}\right) \\
& -\frac{c_{21} e^{c_{3} t+y \sqrt{c_{3} S c}}}{2 c_{3}} \operatorname{erf} c\left(\frac{y \sqrt{\mathrm{Sc}}}{2 \sqrt{t}}+\sqrt{c_{3} t}\right),
\end{aligned}
$$

$V_{4}$

$$
\begin{aligned}
& =\frac{c_{21} e^{c_{1} t-y \sqrt{c_{1} \mathrm{Pr}_{\mathrm{eff}}}}}{2 c_{1}} \operatorname{erf} c\left(\frac{y \sqrt{\mathrm{Pr}_{\mathrm{eff}}}}{2 \sqrt{t}}-\sqrt{c_{1} t}\right) \\
& -\frac{c_{21}}{c_{1}} \operatorname{erf} c\left(\frac{y \sqrt{\mathrm{Pr}_{\mathrm{eff}}}}{2 \sqrt{t}}\right) \\
& +\frac{c_{21} e^{c_{1} t+y \sqrt{c_{1} \mathrm{Pr}_{\mathrm{eff}}}}}{2 c_{1}} \operatorname{erf} c\left(\frac{y \sqrt{\mathrm{Pr}_{\mathrm{eff}}}}{2 \sqrt{t}}+\sqrt{c_{1} t}\right) \\
& -\frac{c_{8} e^{c_{1} t-y \sqrt{c_{1} \mathrm{Pr}_{\mathrm{eff}}}}}{2 c_{1}} \operatorname{erf} c\left(\frac{y \sqrt{\mathrm{Pr}_{\mathrm{eff}}}}{2 \sqrt{t}}-\sqrt{c_{1} t}\right) \\
& +\frac{c_{8}}{c_{1}} \operatorname{erf} c\left(\frac{y \sqrt{\mathrm{Pr}_{\mathrm{eff}}}}{2 \sqrt{t}}\right) \\
& -\frac{c_{8} e^{c_{1} t+y \sqrt{c_{1} \mathrm{Pr}_{\mathrm{eff}}}}}{2 c_{1}} \operatorname{erf} c\left(\frac{y \sqrt{\mathrm{Pr}_{\mathrm{eff}}}}{2 \sqrt{t}}+\sqrt{c_{1} t}\right) \\
& -\frac{c_{9} e^{c_{3} t-y \sqrt{c_{3} \mathrm{Sc}}}}{2 c_{3}} \operatorname{erf} c\left(\frac{y \sqrt{\mathrm{Sc}}}{2 \sqrt{t}}-\sqrt{c_{3} t}\right) \\
& +\frac{c_{9}}{c_{3}} \operatorname{erf} c\left(\frac{y \sqrt{\mathrm{Sc}}}{2 \sqrt{t}}\right) \\
& -\frac{c_{9} e^{c_{3} t+y \sqrt{c_{3} S c}}}{2 c_{3}} \operatorname{erf} c\left(\frac{y \sqrt{\mathrm{Sc}}}{2 \sqrt{t}}+\sqrt{c_{3} t}\right) \text {. }
\end{aligned}
$$

\section{Limiting Cases}

Here some limiting cases are presented.

5.1. Solution in the Absence of Porous Effects $\left(K_{p} \rightarrow 0\right)$. From (21) and (24), it is seen that the temperature fields and concentration fields are not affected by the inverse permeability parameter for the porous medium $K_{p}$. Hence, the velocities for both case of the plate are as

$$
u(y, t)=u_{c}(y, t)+u_{m}(y, t)
$$

where

$$
\begin{aligned}
& u_{c}(y, t)=V_{5}+V_{6}, \\
& V_{5}=c_{2} \int_{0}^{t}\left(\frac{e^{c_{12}(t-s)} \operatorname{erf}\left(\sqrt{c_{12}(t-s)}\right)}{\left(c_{12}\right)^{3 / 2}}-\frac{2 \sqrt{t-s}}{\sqrt{\pi} c_{12}}\right) \\
& \cdot \frac{e^{-M s-y^{2} / 4 s}}{\sqrt{\pi s}} d s-\left[\frac{c_{2}}{\left(c_{12}\right)^{3 / 2} \sqrt{\pi}}\right. \\
& \left.\cdot \int_{0}^{t-1} \frac{e^{c_{12}(t-1-s)-M s-y^{2} / 4 s} \operatorname{erf}\left(\sqrt{c_{12}(t-1-s)}\right)}{\sqrt{s}} d s\right]
\end{aligned}
$$$$
H(t-1)+\left[\frac{c_{2}}{c_{12} \pi}\right.
$$$$
\left.\cdot \int_{0}^{t-1} \frac{(2 \sqrt{t-1-s}) e^{-M s-y^{2} / 4 s}}{\sqrt{s}} d s\right] H(t-1)
$$$$
-c_{7} \int_{0}^{t}\left(\frac{e^{c_{12}(t-s)} \operatorname{erf}\left(\sqrt{c_{12}(t-s)}\right)}{\left(c_{12}\right)^{3 / 2}}-\frac{2 \sqrt{t-s}}{\sqrt{\pi} c_{12}}\right)
$$$$
\cdot \frac{e^{-M s-y^{2} / 4 s}}{\sqrt{\pi s}} d s+\left[\frac{c_{7}}{\left(c_{12}\right)^{3 / 2} \sqrt{\pi}}\right.
$$$$
\left.\cdot \int_{0}^{t-1} \frac{e^{c_{12}(t-1-s)-M s-y^{2} / 4 s} \operatorname{erf}\left(\sqrt{c_{12}(t-1-s)}\right)}{\sqrt{s}} d s\right]
$$$$
H(t-1)-\left[\frac{c_{7}}{c_{12} \pi}\right.
$$$$
\left.\cdot \int_{0}^{t-1} \frac{(2 \sqrt{t-1-s}) e^{-M s-y^{2} / 4 s}}{\sqrt{s}} d s\right] H(t-1)
$$$$
+c_{6} \int_{0}^{t}\left(\frac{e^{c_{13}(t-s)} \operatorname{erf}\left(\sqrt{c_{13}(t-s)}\right)}{\left(c_{13}\right)^{3 / 2}}-\frac{2 \sqrt{t-s}}{\sqrt{\pi} c_{13}}\right)
$$$$
\cdot \frac{e^{-M s-y^{2} / 4 s}}{\sqrt{\pi s}} d s-\left[\frac{c_{6}}{\left(c_{13}\right)^{3 / 2} \sqrt{\pi}}\right.
$$

$$
\left.\cdot \int_{0}^{t-1} \frac{e^{c_{13}(t-1-s)-M s-y^{2} / 4 s} \operatorname{erf}\left(\sqrt{c_{13}(t-1-s)}\right)}{\sqrt{s}} d s\right]
$$

$H(t-1)+\left[\frac{c_{6}}{c_{13} \pi}\right.$

$\left.\cdot \int_{0}^{t-1} \frac{(2 \sqrt{t-1-s}) e^{-M s-y^{2} / 4 s}}{\sqrt{s}} d s\right] H(t-1)$ 


$$
\begin{aligned}
& +c_{4} \int_{0}^{t}\left(e^{c_{13}(t-s)} \operatorname{erf}\left(\sqrt{c_{13}(t-s)}\right)\right) \frac{e^{-M s-y^{2} / 4 s}}{\sqrt{c_{13} \pi s}} d s \\
& +\frac{c_{8}}{c_{12}}\left(t+\frac{\operatorname{Pr}_{\text {eff }} y^{2}}{2}\right) \operatorname{erf} c\left(\frac{y \sqrt{\operatorname{Pr}_{\text {eff }}}}{2 \sqrt{t}}\right) \\
& -\left[\frac{c_{8}}{c_{12}}\left(t-1+\frac{\operatorname{Pr}_{\mathrm{eff}} y^{2}}{2}\right) \operatorname{erf} c\left(\frac{y \sqrt{\mathrm{Pr}_{\mathrm{eff}}}}{2 \sqrt{t-1}}\right)\right] H(t \\
& -1)+\left[\frac{c_{8}}{c_{12}} \frac{y \sqrt{\operatorname{Pr}_{\mathrm{eff}}} \sqrt{t-1}}{\sqrt{\pi}} e^{-y^{2} \operatorname{Pr}_{\mathrm{eff}} / 4(t-1)}\right] H(t-1) \\
& -\frac{c_{9} e^{c_{13} t-y \sqrt{c_{13} \mathrm{Sc}}}}{2 c_{3}} \operatorname{erf} c\left(\frac{y \sqrt{\mathrm{Sc}}}{2 \sqrt{t}}-\sqrt{c_{13} t}\right)+\frac{c_{9}}{c_{13}} \\
& \cdot \operatorname{erf} c\left(\frac{y \sqrt{\mathrm{Sc}}}{2 \sqrt{t}}\right)-\frac{c_{10} e^{c_{13} t-y \sqrt{\mathrm{Scc}_{3}}}}{2 c_{3}^{2}} \\
& \cdot \operatorname{erf} c\left(\frac{y \sqrt{\mathrm{Sc}}}{2 \sqrt{t}}-\sqrt{c_{13} t}\right)-\frac{c_{9} e^{c_{13} t+y \sqrt{c_{13} \mathrm{Sc}}}}{2 c_{13}} \\
& \cdot \operatorname{erf} c\left(\frac{y \sqrt{\mathrm{Sc}}}{2 \sqrt{t}}+\sqrt{c_{13} t}\right) \\
& V_{6}=\frac{c_{10}}{c_{13}}\left(t+\frac{\mathrm{Sc} y^{2}}{2}\right) \operatorname{erf} c\left(\frac{y \sqrt{\mathrm{Sc}}}{2 \sqrt{t}}\right)-\left[\frac{c_{10}}{c_{13}^{2}}\right. \\
& \left.\cdot \operatorname{erf} c\left(\frac{y \sqrt{\mathrm{Sc}}}{2 \sqrt{t-1}}\right)\right] H(t-1) \\
& -\frac{\left(c_{8}-c_{11}\right) e^{c_{12} t-y \sqrt{\mathrm{Pr}_{\mathrm{eff}} c_{12}}}}{2 c_{12}^{2}} \operatorname{erf} c\left(\frac{y \sqrt{\mathrm{Pr}_{\mathrm{eff}}}}{2 \sqrt{t}}-\sqrt{c_{12} t}\right) \\
& -\frac{\left(c_{8}-c_{11}\right) e^{c_{12} t+y \sqrt{\mathrm{Pr}_{\mathrm{eff}} c_{12}}}}{2 c_{12}^{2}} \operatorname{erf} c\left(\frac{y \sqrt{\mathrm{Pr}_{\mathrm{eff}}}}{2 \sqrt{t}}+\sqrt{c_{12} t}\right) \\
& +\left[\frac{c_{10}}{c_{13}} \frac{y \sqrt{\mathrm{Sc}} \sqrt{t-1}}{\sqrt{\pi}} e^{-y^{2} \mathrm{Sc} / 4(t-1)}\right] H(t-1)-\frac{c_{10}}{c_{13}} \\
& \cdot \frac{y \sqrt{\mathrm{Sc}} \sqrt{t}}{\sqrt{\pi}} e^{-y^{2} \mathrm{Sc} / 4 t}+\frac{c_{10}}{c_{13}^{2}} \operatorname{erf} c\left(\frac{y \sqrt{\mathrm{Sc}}}{2 \sqrt{t}}\right) \\
& -\frac{\left(c_{8}-c_{11}\right)}{c_{12}} \frac{y \sqrt{\operatorname{Pr}_{\mathrm{eff}}} \sqrt{t}}{\sqrt{\pi}} e^{-y^{2} \operatorname{Pr}_{\mathrm{eff}} / 4 t}+\frac{\left(c_{8}-c_{11}\right)}{c_{12}^{2}} \\
& \cdot \operatorname{erf} c\left(\frac{y \sqrt{\operatorname{Pr}_{\text {eff }}}}{2 \sqrt{t}}\right)-\left[\frac{\left(c_{8}-c_{11}\right)}{c_{12}^{2}} \operatorname{erf} c\left(\frac{y \sqrt{\operatorname{Pr}_{\mathrm{eff}}}}{2 \sqrt{t-1}}\right)\right] \\
& \cdot H(t-1)+\left[\frac{c_{10} e^{c_{13}(t-1)-y \sqrt{\mathrm{Scc}_{3}}}}{2 c_{13}^{2}}\right. \\
& \left.\cdot \operatorname{erf} c\left(\frac{y \sqrt{\mathrm{Sc}}}{2 \sqrt{(t-1)}}-\sqrt{c_{13}(t-1)}\right)\right] H(t-1) \\
& -\frac{c_{10} e^{c_{13} t+y \sqrt{\mathrm{Scc}_{3}}}}{2 c_{13}^{2}} \operatorname{erf} c\left(\frac{y \sqrt{\mathrm{Sc}}}{2 \sqrt{t}}+\sqrt{c_{13} t}\right)
\end{aligned}
$$$$
-\left[\frac{c_{10}}{c_{13}}\left(t-1+\frac{\mathrm{Sc} y^{2}}{2}\right) \operatorname{erf} c\left(\frac{y \sqrt{\mathrm{Sc}}}{2 \sqrt{t-1}}\right)\right] H(t
$$$$
-1)+\left[\frac{\left(c_{8}-c_{11}\right) e^{c_{12}(t-1)-y \sqrt{\operatorname{Pr}_{\mathrm{eff}} c_{12}}}}{2 c_{12}^{2}}\right.
$$$$
\left.\cdot \operatorname{erf} c\left(\frac{y \sqrt{\mathrm{Pr}_{\mathrm{eff}}}}{2 \sqrt{(t-1)}}-\sqrt{c_{12}(t-1)}\right)\right] H(t-1)
$$$$
+\left[\frac{\left(c_{8}-c_{11}\right) e^{c_{12}(t-1)+y \sqrt{\operatorname{Pr}_{\text {eff }} c_{12}}}}{2 c_{12}^{2}}\right.
$$$$
\left.\cdot \operatorname{erf} c\left(\frac{y \sqrt{\mathrm{Pr}_{\mathrm{eff}}}}{2 \sqrt{(t-1)}}+\sqrt{c_{12}(t-1)}\right)\right] H(t-1) \text {, }
$$

and for isothermal

$$
\begin{aligned}
& u_{c}(y, t)=V_{7}+V_{8}, \\
& V_{7} \\
& =c_{2} \int_{0}^{t}\left(e^{c_{12}(t-s)} \operatorname{erf}\left(\sqrt{c_{12}(t-s)}\right)\right) \frac{e^{-M s-y^{2} / 4 s}}{\sqrt{c_{1} \pi s}} d s \\
& +c_{4} \int_{0}^{t}\left(e^{c_{13}(t-s)} \operatorname{erf}\left(\sqrt{c_{13}(t-s)}\right)\right) \frac{e^{-M s-y^{2} / 4 s}}{\sqrt{c_{13} \pi s}} d s \\
& +c_{19} \int_{0}^{t}\left(e^{c_{13}(t-s)} \operatorname{erf}\left(\sqrt{c_{13}(t-s)}\right)\right) \frac{e^{-M s-y^{2} / 4 s}}{\sqrt{c_{13} \pi s}} d s \\
& \quad-c_{20} \int_{0}^{t}\left(e^{c_{12}(t-s)} \operatorname{erf}\left(\sqrt{c_{12}(t-s)}\right)\right) \frac{e^{-M s-y^{2} / 4 s}}{\sqrt{c_{12} \pi s}} d s \\
& \quad-\frac{c_{21} e^{c_{13} t-y \sqrt{c_{13} \mathrm{Sc}}}}{2 c_{13}} \operatorname{erf}\left(\frac{y \sqrt{\mathrm{Sc}}}{2 \sqrt{t}}-\sqrt{c_{13} t}\right) \\
& +\frac{c_{21}}{c_{13}} \operatorname{erf} c\left(\frac{y \sqrt{\mathrm{Sc}}}{2 \sqrt{t}}\right) \\
& +\frac{c_{21} e^{c_{13} t+y \sqrt{c_{13} \mathrm{Sc}}}}{2 c_{13}} \operatorname{erf} c\left(\frac{y \sqrt{\mathrm{Sc}}}{2 \sqrt{t}}+\sqrt{c_{13} t}\right)
\end{aligned}
$$

$V_{8}$

$$
\begin{aligned}
= & \frac{c_{21} e^{c_{12} t-y \sqrt{c_{12} \operatorname{Pr}_{\text {eff }}}}}{2 c_{12}} \operatorname{erf} c\left(\frac{y \sqrt{\operatorname{Pr}_{\text {eff }}}}{2 \sqrt{t}}-\sqrt{c_{12} t}\right) \\
& -\frac{c_{21}}{c_{12}} \operatorname{erf} c\left(\frac{y \sqrt{\operatorname{Pr}_{\text {eff }}}}{2 \sqrt{t}}\right) \\
& +\frac{c_{21} e^{c_{12} t+y \sqrt{c_{12} \operatorname{Pr}_{\text {eff }}}}}{2 c_{12}} \operatorname{erf} c\left(\frac{y \sqrt{\operatorname{Pr}_{\text {eff }}}}{2 \sqrt{t}}+\sqrt{c_{12} t}\right) \\
& -\frac{c_{8} e^{c_{12} t-y \sqrt{c_{12} \operatorname{Pr}_{\text {eff }}}}}{2 c_{12}} \operatorname{erf} c\left(\frac{y \sqrt{\operatorname{Pr}_{\text {eff }}}}{2 \sqrt{t}}-\sqrt{c_{12} t}\right)
\end{aligned}
$$




$$
\begin{aligned}
& +\frac{c_{8}}{c_{12}} \operatorname{erf} c\left(\frac{y \sqrt{\mathrm{Pr}_{\mathrm{eff}}}}{2 \sqrt{t}}\right) \\
& -\frac{c_{8} e^{c_{12} t+y \sqrt{c_{12} \mathrm{Pr}_{\mathrm{eff}}}}}{2 c_{12}} \operatorname{erf} c\left(\frac{y \sqrt{\mathrm{Pr}_{\mathrm{eff}}}}{2 \sqrt{t}}+\sqrt{c_{12} t}\right) \\
& -\frac{c_{9} e^{c_{13} t-y \sqrt{c_{13} \mathrm{Sc}}}}{2 c_{13}} \operatorname{erf} c\left(\frac{y \sqrt{\mathrm{Sc}}}{2 \sqrt{t}}-\sqrt{c_{13} t}\right) \\
& +\frac{c_{9}}{c_{13}} \operatorname{erf} c\left(\frac{y \sqrt{\mathrm{Sc}}}{2 \sqrt{t}}\right) \\
& -\frac{c_{9} e^{c_{13} t+y \sqrt{c_{13} \mathrm{Sc}}}}{2 c_{13}} \operatorname{erf} c\left(\frac{y \sqrt{\mathrm{Sc}}}{2 \sqrt{t}}+\sqrt{c_{13} t}\right) .
\end{aligned}
$$

5.2. Solutions in the Absence of Free Convection. Consider that the fluid flow is due to bounding plate and the corresponding $\mathrm{Gr}$ and $\mathrm{Gm}$ are zero. In this case, the fluid motion is only by the mechanical part of velocities given by (31).

5.3. Solutions in the Absence of Mechanical Effects. Let us suppose that the infinite plate is motionless at every time; that is, $f(t)$ is zero for all values of $t$. Mechanical parts are equivalently zero in both cases of the plates. Therefore, the motion in the fluid is a result of the free convection which is caused due to the buoyancy forces. Hence, the fluid velocities are only represented by their convective parts obtained in (28) and (36).

5.4. Solution in the Absence of Magnetic Parameter $(M \rightarrow 0)$. From (21) and (24), the temperature fields and concentration fields are not affected by $M$, and the velocities in the absence of $M$ are given by

$$
u(y, t)=u_{c}(y, t)+u_{m}(y, t)
$$

where

$$
\begin{aligned}
& u_{c}(y, t)=V_{9}+V_{10}, \\
& V_{9}=c_{2} \int_{0}^{t}\left(\frac{e^{c_{15}(t-s)} \operatorname{erf}\left(\sqrt{c_{15}(t-s)}\right)}{\left(c_{15}\right)^{3 / 2}}-\frac{2 \sqrt{t-s}}{\sqrt{\pi} c_{15}}\right) \\
& \cdot \frac{e^{-K_{p} s-y^{2} / 4 s}}{\sqrt{\pi s}} d s-\left[\frac{c_{2}}{\left(c_{15}\right)^{3 / 2} \sqrt{\pi}}\right. \\
& \left.\cdot \int_{0}^{t-1} \frac{e^{c_{15}(t-1-s)-K_{p} s-y^{2} / 4 s} \operatorname{erf}\left(\sqrt{c_{15}(t-1-s)}\right)}{\sqrt{s}} d s\right] \\
& \cdot H(t-1)+\left[\frac{c_{2}}{c_{15} \pi}\right. \\
& \left.\cdot \int_{0}^{t-1} \frac{(2 \sqrt{t-1-s}) e^{-K_{p} s-y^{2} / 4 s}}{\sqrt{s}} d s\right] H(t-1)
\end{aligned}
$$

$$
\begin{aligned}
& -c_{7} \int_{0}^{t}\left(\frac{e^{c_{15}(t-s)} \operatorname{erf}\left(\sqrt{c_{15}(t-s)}\right)}{\left(c_{15}\right)^{3 / 2}}-\frac{2 \sqrt{t-s}}{\sqrt{\pi} c_{15}}\right) \\
& \cdot \frac{e^{-K_{p} s-y^{2} / 4 s}}{\sqrt{\pi s}} d s+\left[\frac{c_{7}}{\left(c_{15}\right)^{3 / 2} \sqrt{\pi}}\right. \\
& \left.\cdot \int_{0}^{t-1} \frac{e^{c_{15}(t-1-s)-K_{p} s-y^{2} / 4 s} \operatorname{erf}\left(\sqrt{c_{15}(t-1-s)}\right)}{\sqrt{s}} d s\right]
\end{aligned}
$$$$
H(t-1)-\left[\frac{c_{7}}{c_{15} \pi}\right.
$$$$
\left.\int_{0}^{t-1} \frac{(2 \sqrt{t-1-s}) e^{-K_{p} s-y^{2} / 4 s}}{\sqrt{s}} d s\right] H(t-1)
$$$$
+c_{6} \int_{0}^{t}\left(\frac{e^{c_{16}(t-s)} \operatorname{erf}\left(\sqrt{c_{16}(t-s)}\right)}{\left(c_{16}\right)^{3 / 2}}-\frac{2 \sqrt{t-s}}{\sqrt{\pi} c_{16}}\right)
$$$$
\cdot \frac{e^{-K_{p} s-y^{2} / 4 s}}{\sqrt{\pi s}} d s-\left[\frac{c_{6}}{\left(c_{16}\right)^{3 / 2} \sqrt{\pi}}\right.
$$$$
\left.\cdot \int_{0}^{t-1} \frac{e^{c_{16}(t-1-s)-K_{p} s-y^{2} / 4 s} \operatorname{erf}\left(\sqrt{c_{16}(t-1-s)}\right)}{\sqrt{s}} d s\right]
$$$$
H(t-1)+\left[\frac{c_{6}}{c_{16} \pi}\right.
$$$$
\left.\cdot \int_{0}^{t-1} \frac{(2 \sqrt{t-1-s}) e^{-K_{p} s-y^{2} / 4 s}}{\sqrt{s}} d s\right] H(t-1)
$$$$
+c_{4} \int_{0}^{t}\left(e^{c_{16}(t-s)} \operatorname{erf}\left(\sqrt{c_{16}(t-s)}\right)\right) \frac{e^{-K_{p} s-y^{2} / 4 s}}{\sqrt{c_{16} \pi s}} d s
$$$$
+\frac{c_{8}}{c_{15}}\left(t+\frac{\operatorname{Pr}_{\text {eff }} y^{2}}{2}\right) \operatorname{erf} c\left(\frac{y \sqrt{\operatorname{Pr}_{\text {eff }}}}{2 \sqrt{t}}\right)
$$$$
-\left[\frac{c_{8}}{c_{15}}\left(t-1+\frac{\operatorname{Pr}_{\mathrm{eff}} y^{2}}{2}\right) \operatorname{erf} c\left(\frac{y \sqrt{\mathrm{Pr}_{\mathrm{eff}}}}{2 \sqrt{t-1}}\right)\right] H(t
$$$$
-1)+\left[\frac{c_{8}}{c_{15}} \frac{y \sqrt{\mathrm{Pr}_{\mathrm{eff}}} \sqrt{t-1}}{\sqrt{\pi}} e^{-y^{2} \mathrm{Pr}_{\mathrm{eff}} / 4(t-1)}\right] H(t-1)
$$$$
-\frac{c_{9} e^{c_{16} t-y \sqrt{c_{16} \mathrm{Sc}}}}{2 c_{16}} \operatorname{erf} c\left(\frac{y \sqrt{\mathrm{Sc}}}{2 \sqrt{t}}-\sqrt{c_{16} t}\right)+\frac{c_{9}}{c_{16}}
$$$$
\cdot \operatorname{erf} c\left(\frac{y \sqrt{\mathrm{Sc}}}{2 \sqrt{t}}\right)-\frac{c_{10} e^{c_{16} t-y \sqrt{\mathrm{Scc}_{16}}}}{2 c_{16}^{2}}
$$$$
\cdot \operatorname{erf} c\left(\frac{y \sqrt{\mathrm{Sc}}}{2 \sqrt{t}}-\sqrt{c_{16} t}\right)-\frac{c_{9} e^{c_{16} t+y \sqrt{c_{16} \mathrm{Sc}}}}{2 c_{16}}
$$ 


$$
\begin{aligned}
& \cdot \operatorname{erf} c\left(\frac{y \sqrt{\mathrm{Sc}}}{2 \sqrt{t}}+\sqrt{c_{16} t}\right) \\
& V_{10}=\frac{c_{10}}{c_{16}}\left(t+\frac{\mathrm{Sc} y^{2}}{2}\right) \operatorname{erf} c\left(\frac{y \sqrt{\mathrm{Sc}}}{2 \sqrt{t}}\right)-\left[\frac{c_{10}}{c_{3}^{2}}\right. \\
& \left.\cdot \operatorname{erf} c\left(\frac{y \sqrt{\mathrm{Sc}}}{2 \sqrt{t-1}}\right)\right] H(t-1) \\
& -\frac{\left(c_{8}-c_{11}\right) e^{c_{15} t-y \sqrt{\mathrm{Pr}_{\mathrm{eff}} c_{15}}}}{2 c_{15}^{2}} \operatorname{erf} c\left(\frac{y \sqrt{\mathrm{Pr}_{\mathrm{eff}}}}{2 \sqrt{t}}-\sqrt{c_{15} t}\right) \\
& -\frac{\left(c_{8}-c_{11}\right) e^{c_{1} t+y \sqrt{\mathrm{Pr}_{\mathrm{eff}} c_{1}}}}{2 c_{15}^{2}} \operatorname{erf} c\left(\frac{y \sqrt{\operatorname{Pr}_{\text {eff }}}}{2 \sqrt{t}}+\sqrt{c_{1} t}\right) \\
& +\left[\frac{c_{10}}{c_{16}} \frac{y \sqrt{S c} \sqrt{t-1}}{\sqrt{\pi}} e^{-y^{2} \mathrm{Sc} / 4(t-1)}\right] H(t-1)-\frac{c_{10}}{c_{16}} \\
& \cdot \frac{y \sqrt{\mathrm{Sc}} \sqrt{t}}{\sqrt{\pi}} e^{-y^{2} \mathrm{Sc} / 4 t}+\frac{c_{10}}{c_{16}^{2}} \operatorname{erf} c\left(\frac{y \sqrt{\mathrm{Sc}}}{2 \sqrt{t}}\right) \\
& -\frac{\left(c_{8}-c_{11}\right)}{c_{15}} \frac{y \sqrt{\mathrm{Pr}_{\mathrm{eff}}} \sqrt{t}}{\sqrt{\pi}} e^{-y^{2} \operatorname{Pr}_{\mathrm{eff}} / 4 t}+\frac{\left(c_{8}-c_{11}\right)}{c_{15}^{2}} \\
& \cdot \operatorname{erf} c\left(\frac{y \sqrt{\operatorname{Pr}_{\text {eff }}}}{2 \sqrt{t}}\right)-\left[\frac{\left(c_{8}-c_{11}\right)}{c_{15}^{2}} \operatorname{erf} c\left(\frac{y \sqrt{\operatorname{Pr}_{\text {eff }}}}{2 \sqrt{t-1}}\right)\right] \\
& \cdot H(t-1)+\left[\frac{c_{10} e^{c_{3}(t-1)-y \sqrt{\mathrm{Scc}_{3}}}}{2 c_{16}^{2}}\right. \\
& \left.\cdot \operatorname{erf} c\left(\frac{y \sqrt{\mathrm{Sc}}}{2 \sqrt{(t-1)}}-\sqrt{c_{3}(t-1)}\right)\right] H(t-1) \\
& -\frac{c_{10} e^{c_{16} t+y \sqrt{\mathrm{Scc}_{16}}}}{2 c_{16}^{2}} \operatorname{erf} c\left(\frac{y \sqrt{\mathrm{Sc}}}{2 \sqrt{t}}+\sqrt{c_{16} t}\right) \\
& -\left[\frac{c_{10}}{c_{16}}\left(t-1+\frac{\mathrm{Sc} y^{2}}{2}\right) \operatorname{erf} c\left(\frac{y \sqrt{\mathrm{Sc}}}{2 \sqrt{t-1}}\right)\right] H(t \\
& -1)+\left[\frac{\left(c_{8}-c_{11}\right) e^{c_{15}(t-1)-y \sqrt{\mathrm{Pr}_{\mathrm{eff}} c_{15}}}}{2 c_{15}^{2}}\right. \\
& \left.\cdot \operatorname{erf} c\left(\frac{y \sqrt{\operatorname{Pr}_{\text {eff }}}}{2 \sqrt{(t-1)}}-\sqrt{c_{15}(t-1)}\right)\right] H(t-1) \\
& +\left[\frac{\left(c_{8}-c_{11}\right) e^{c_{15}(t-1)+y \sqrt{\mathrm{Pr}_{\mathrm{eff}} c_{15}}}}{2 c_{15}^{2}}\right. \\
& \left.\cdot \operatorname{erf} c\left(\frac{y \sqrt{\operatorname{Pr}_{\text {eff }}}}{2 \sqrt{(t-1)}}+\sqrt{c_{15}(t-1)}\right)\right] H(t-1), \\
& u_{c}(y, t)=V_{11}+V_{12} \text {, } \\
& V_{11} \\
& =c_{2} \int_{0}^{t}\left(e^{c_{15}(t-s)} \operatorname{erf}\left(\sqrt{c_{15}(t-s)}\right)\right) \frac{e^{-K_{p} s-y^{2} / 4 s}}{\sqrt{c_{15} \pi s}} d s \\
& +c_{4} \int_{0}^{t}\left(e^{c_{16}(t-s)} \operatorname{erf}\left(\sqrt{c_{16}(t-s)}\right)\right) \frac{e^{-K_{p} s-y^{2} / 4 s}}{\sqrt{c_{16} \pi s}} d s \\
& +c_{19} \int_{0}^{t}\left(e^{c_{16}(t-s)} \operatorname{erf}\left(\sqrt{c_{16}(t-s)}\right)\right) \frac{e^{-K_{p} s-y^{2} / 4 s}}{\sqrt{c_{16} \pi s}} d s \\
& -c_{20} \int_{0}^{t}\left(e^{c_{15}(t-s)} \operatorname{erf}\left(\sqrt{c_{15}(t-s)}\right)\right) \frac{e^{-K_{p} s-y^{2} / 4 s}}{\sqrt{c_{15} \pi s}} d s \\
& -\frac{c_{21} e^{c_{16} t-y \sqrt{c_{16} \mathrm{Sc}}}}{2 c_{16}} \operatorname{erf} c\left(\frac{y \sqrt{\mathrm{Sc}}}{2 \sqrt{t}}-\sqrt{c_{16} t}\right) \\
& +\frac{c_{21}}{c_{16}} \operatorname{erf} c\left(\frac{y \sqrt{\mathrm{Sc}}}{2 \sqrt{t}}\right) \\
& -\frac{c_{21} e^{c_{16} t+y \sqrt{c_{16} \mathrm{Sc}}}}{2 c_{16}} \operatorname{erf} c\left(\frac{y \sqrt{\mathrm{Sc}}}{2 \sqrt{t}}+\sqrt{c_{16} t}\right), \\
& =\frac{c_{21} e^{c_{15} t-y \sqrt{c_{15} \mathrm{Pr}_{\text {eff }}}}}{2 c_{15}} \operatorname{erf} c\left(\frac{y \sqrt{\operatorname{Pr}_{\text {eff }}}}{2 \sqrt{t}}-\sqrt{c_{15} t}\right) \\
& -\frac{c_{21}}{c_{15}} \operatorname{erf} c\left(\frac{y \sqrt{\operatorname{Pr}_{\text {eff }}}}{2 \sqrt{t}}\right) \\
& +\frac{c_{21} e^{c_{15} t+y \sqrt{c_{15} \mathrm{Pr}_{\mathrm{eff}}}}}{2 c_{15}} \operatorname{erf} c\left(\frac{y \sqrt{\mathrm{Pr}_{\mathrm{eff}}}}{2 \sqrt{t}}+\sqrt{c_{15} t}\right) \\
& -\frac{c_{8} e^{c_{15} t-y \sqrt{c_{15} \mathrm{Pr}_{\mathrm{eff}}}}}{2 c_{15}} \operatorname{erf} c\left(\frac{y \sqrt{\operatorname{Pr}_{\mathrm{eff}}}}{2 \sqrt{t}}-\sqrt{c_{15} t}\right) \\
& +\frac{c_{8}}{c_{15}} \operatorname{erf} c\left(\frac{y \sqrt{\operatorname{Pr}_{\text {eff }}}}{2 \sqrt{t}}\right) \\
& -\frac{c_{8} e^{c_{15} t+y \sqrt{c_{15} \mathrm{Pr}_{\mathrm{eff}}}}}{2 c_{15}} \operatorname{erf} c\left(\frac{y \sqrt{\operatorname{Pr}_{\mathrm{eff}}}}{2 \sqrt{t}}+\sqrt{c_{15} t}\right) \\
& -\frac{c_{9} e^{c_{16} t-y \sqrt{c_{16} \mathrm{Sc}}}}{2 c_{16}} \operatorname{erf} c\left(\frac{y \sqrt{\mathrm{Sc}}}{2 \sqrt{t}}-\sqrt{c_{16} t}\right) \\
& +\frac{c_{9}}{c_{16}} \operatorname{erf} c\left(\frac{y \sqrt{\mathrm{Sc}}}{2 \sqrt{t}}\right) \\
& -\frac{c_{9} e^{c_{16} t+y \sqrt{c_{16} \mathrm{Sc}}}}{2 c_{16}} \operatorname{erf} c\left(\frac{y \sqrt{\mathrm{Sc}}}{2 \sqrt{t}}+\sqrt{c_{16} t}\right) .
\end{aligned}
$$




\section{Special Cases}

The obtained velocities in Section 3 are more general. Hence, some special cases of the present results are presented. In order to learn much more about the physical importance of the problem, technical relevance of these cases is found in literature.

6.1. Case-I: $f(t)=f H(t)$. Consider $f(t)=f H(t)$, where $f$ and $H(\cdot)$ are a dimensionless constant and the unit step function. The infinite vertical plate applies a constant shear stress to the fluid which is observed after time $t=0$. There is no change seen in convective part of the velocity, while the mechanical part has been changed as

$$
u_{m}(y, t)=-\frac{f}{\sqrt{\pi}} \int_{0}^{t} \frac{e^{-y^{2} / 4 s-H_{1} s}}{\sqrt{s}} d s
$$

equivalently

$$
u_{m}(y, t)=-\frac{f}{\sqrt{H_{1}}} e^{-y \sqrt{H_{1}}}+\frac{2 f}{\sqrt{\pi}} \int_{\sqrt{t}}^{\infty} e^{-y^{2} / 4 z^{2}-H_{1} z^{2}} d z
$$

for $K_{p} \neq 0, M \neq 0$. Moreover, if we take $M=0$, (45) reduces to the form

$$
u_{m}(y, t)=-\frac{f}{\sqrt{K_{p}}} e^{-y \sqrt{K_{p}}}+\frac{2 f}{\sqrt{\pi}} \int_{\sqrt{t}}^{\infty} e^{-y^{2} / 4 z^{2}-K_{p} z^{2}} d z
$$

which is equivalent to [30, Equation (28)] with the correction of $\sqrt{K_{p}}$.

Moreover, in the absence of both $K_{p}$ and $M$, (45) is

$$
u_{m}(y, t)=-\frac{f}{\sqrt{\pi}} \int_{0}^{t} \frac{e^{-y^{2} / 4 s}}{\sqrt{s}} d s
$$

6.2. Case-II: $f(t)=f \sin (\omega t)$. We take $f(t)=f \sin (\omega t)$ in which an oscillating shear stress to the fluid is applied by the plate, where $\omega$ denotes the dimensionless frequency of the shear stress. The convective part of velocity remains the same like the first case; however the mechanical part changed and takes the form

$$
u_{m}(y, t)=-\frac{f}{\sqrt{\pi}} \int_{0}^{t} \frac{\sin (\omega t-\omega s) e^{-y^{2} / 4 s-H_{1} s}}{\sqrt{s}} d s .
$$

Furthermore, it can be written as a sum of the steady-state and transient solutions

$$
u_{m}(y, t)=u_{m s}(y, t)+u_{m t}(y, t),
$$

where

$$
\begin{aligned}
& u_{m s}(y, t)=-\frac{f}{\sqrt{\pi}} \int_{0}^{t} \frac{\sin (\omega t-\omega s) e^{-y^{2} / 4 s-H_{1} s}}{\sqrt{s}} d s, \\
& u_{m t}(y, t)=\frac{f}{\sqrt{\pi}} \int_{t}^{\infty} \frac{\sin (\omega t-\omega s) e^{-y^{2} / 4 s-H_{1} s}}{\sqrt{s}} d s .
\end{aligned}
$$

By taking $M=0$, the steady-state component reduces to [30, Equation (35)]

$$
u_{m s}(y, t)=-\frac{f}{\sqrt{\pi}} \int_{0}^{t} \frac{\sin (\omega t-\omega s) e^{-y^{2} / 4 s-K_{p} s}}{\sqrt{s}} d s .
$$

In addition, when $K_{p}=0$, (52) results in

$$
u_{m s}(y, t)=-\frac{f}{\sqrt{\pi}} \int_{0}^{t} \frac{\sin (\omega t-\omega s) e^{-y^{2} / 4 s}}{\sqrt{s}} d s,
$$

which can be written in simplified form as

$$
\begin{aligned}
& u_{m s}(y, t) \\
& =\frac{f}{\sqrt{\omega}} \exp \left(-y \sqrt{\frac{\omega}{2}}\right) \cos \left(\omega t-y \sqrt{\frac{\omega}{2}}+\frac{\pi}{4}\right),
\end{aligned}
$$

equivalent to [26, Equation (33)].

\section{Graphical Results and Discussion}

In this section the obtained solutions are numerically studied for determining the effects of several involved parameters such as magnetic parameter $M$, the inverse permeability parameter for the porous medium $K_{p}$, effective Prandtl number $\operatorname{Pr}_{\text {eff }}$, Grashof number Gr, modified Grashof number Gm, Schmidt number Sc, shear stress $f$, and Soret number Sr. It is depicted from Figure 2 that, with increasing values of $\mathrm{Gr}$, the velocity profiles increase. Physically, this scenario is important due to the fact that an increase in $\mathrm{Gr}$ gives rise to buoyancy effects which results in more induced flows. In Figure 3 the velocity profiles for different values of modified Grashof number Gm are displayed. It can be seen that the velocity and boundary layer thickness decrease as with increasing distance from the leading edge. However, the velocity and boundary layer thickness increase with increasing values of $\mathrm{Gm}$. In Figure 4 the velocity profiles are shown for different values of Schmidt number Sc. Here the values of Sc are chosen as $0.22,0.60$, and 0.96 to represent the presence of species by hydrogen, water vapor, and carbon dioxide, respectively. It is observed that the velocity decreases with increasing Schmidt number. Physically, this refers to the phenomenon that increasing Schmidt number implies the dominance of the viscous forces over the diffusional effects. As a result, the flow will be therefore decelerated with a rise in Schmidt number. The velocity profiles for different values of magnetic parameter $M$ are presented in Figure 5. Magnetic field ranges from 0 to 2 . Velocity is decreasing with increasing values of $M$. Physically, it is true because the frictional force is directly proportional to $M$; therefore fluid flow tends to resist and decrease in velocity is observed. It is further observed that, in the absence of the magnetic field, the MHD effect approaches zero and hydrodynamic flow is observed. The effects of inverse permeability parameter $K_{p}$ on the velocity profiles are presented in Figure 6 . It is found that velocity decreases with increasing $K_{p}$. This result is in good agreement with the previous study [30], Figure 3. The effects of the wall shear stress $f$ are shown in Figure 7. 


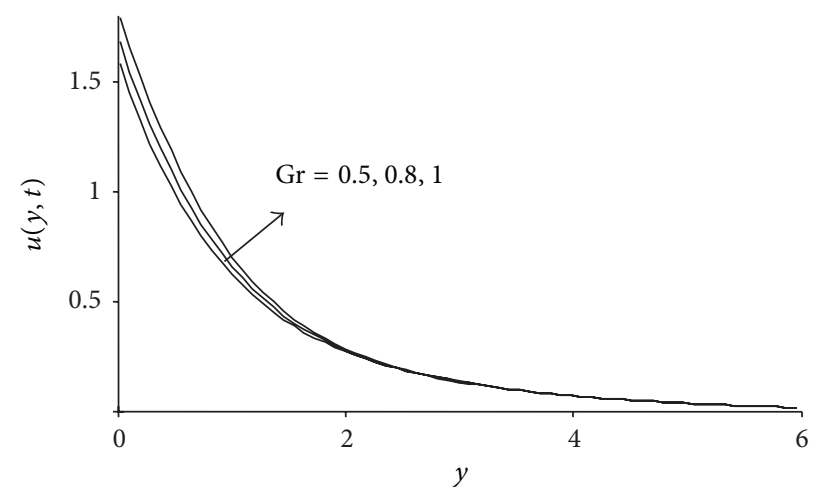

FIGURE 2: Velocity profiles for $\operatorname{Pr}_{\text {eff }}=0.350\left(N_{r}=1, \operatorname{Pr}=0.7\right), K_{p}=0.7, t=1.2$, and different values of Gr when the plate applies constant shear stress $f=-0.25$.

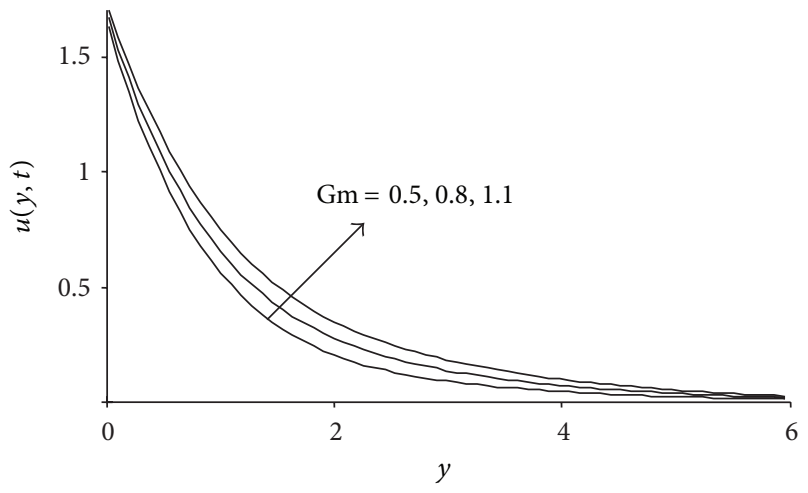

FIGURE 3: Velocity profiles for $\operatorname{Pr}_{\text {eff }}=0.350\left(N_{r}=1, \operatorname{Pr}=0.7\right)$, $K_{p}=0.7, t=1.2$, and different values of $\mathrm{Gm}$ when the plate applies constant shear stress $f=-0.25$.

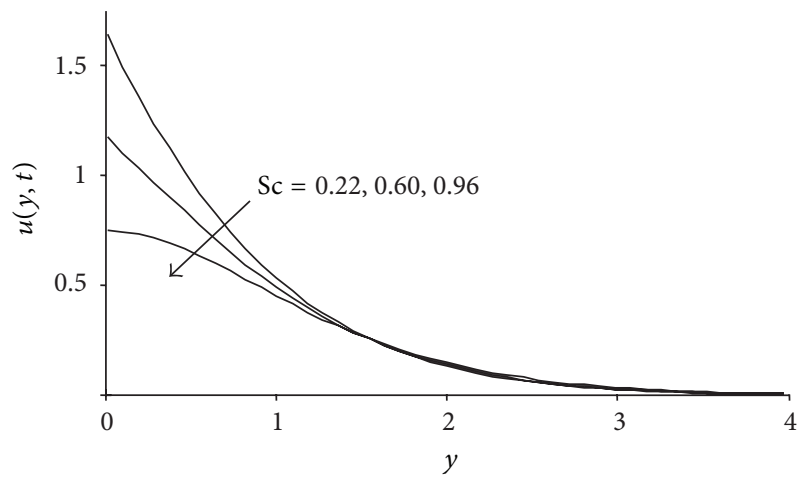

Figure 4: Velocity profiles for $\operatorname{Pr}_{\text {eff }}=0.350\left(N_{r}=1, \operatorname{Pr}=0.7\right)$, $K_{p}=0.7, t=1.2$, and different values of Sc when the plate applies constant shear stress $f=-0.25$.

The velocity of fluid is found to decrease with increasing $f$. The influence of the effective Prandtl number $\operatorname{Pr}_{\text {eff }}$ on velocity profiles are presented in Figure 8. It is observed that the velocity is a decreasing function with respect to $\operatorname{Pr}_{\text {eff }}$. These graphical results are in accordance with [30], Figure 2. The velocity profiles for different values of Soret number $\mathrm{Sr}$ are shown in Figure 9. It is noticed that the velocity increases with

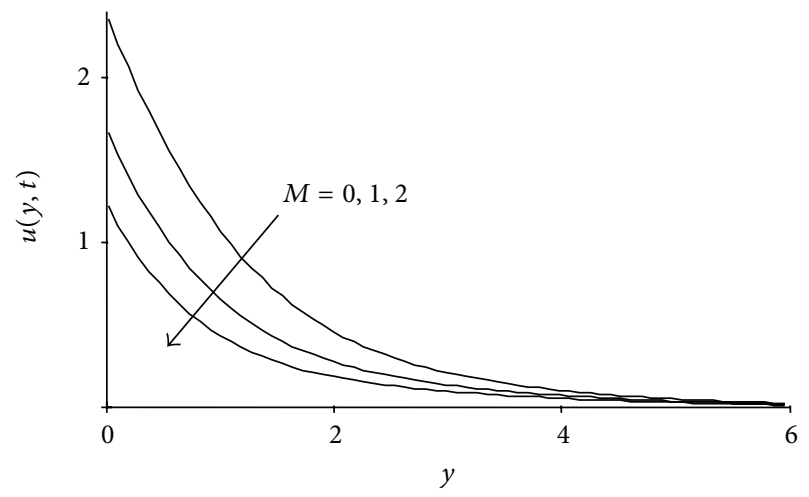

FIGURE 5: Velocity profiles for $\operatorname{Pr}_{\text {eff }}=0.350\left(N_{r}=1, \operatorname{Pr}=0.7\right)$, $K_{p}=0.7, t=1.2$, and different values of $M$ when the plate applies constant shear stress $f=-0.25$.

increasing values of Sr. The temperature variations against $y$ for various values of effective Prandtl number are shown in Figure 10. The significant decrease of the temperature is found as a result of an increase of the effective Prandtl number. The fluid temperature is maximum at the boundary while it has minimum value as far from the plate. The concentration profiles for variuos values of Schmidt number Sc are drawn in Figure 11. It is clear from this figure that the concentration profiles and the concentration boundary layer thickness decrease with increasing values of Sc. Physically, it is valid as molecular diffusivity decreases while increasing the value Sc.

\section{Conclusions}

The aim of this research is to examine the unsteady MHD free convection flow of an incompressible Newtonian fluid over an infinite plate. The ramped wall temperature, thermal diffusion, and wall shear stress to the fluid are taken. Closedform solutions for velocity field, temperature field, and concentration field are obtained using the Laplace transform technique. The results are shown in terms of the complementary error function. The initial and boundary conditions are satisfied by the obtained results and different parameters 


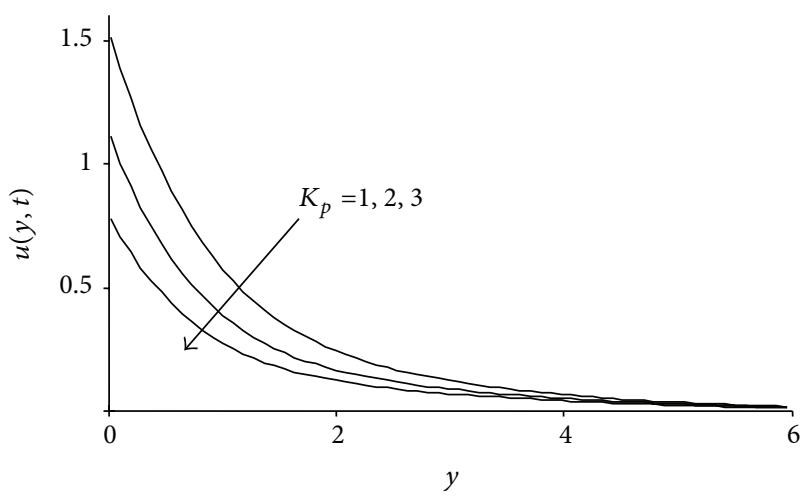

FIgURE 6: Velocity profiles for $\operatorname{Pr}_{\text {eff }}=0.350\left(N_{r}=1, \operatorname{Pr}=0.7\right)$, $t=1.2$, and different values of $K_{p}$ when the plate applies constant shear stress $f=-0.25$.

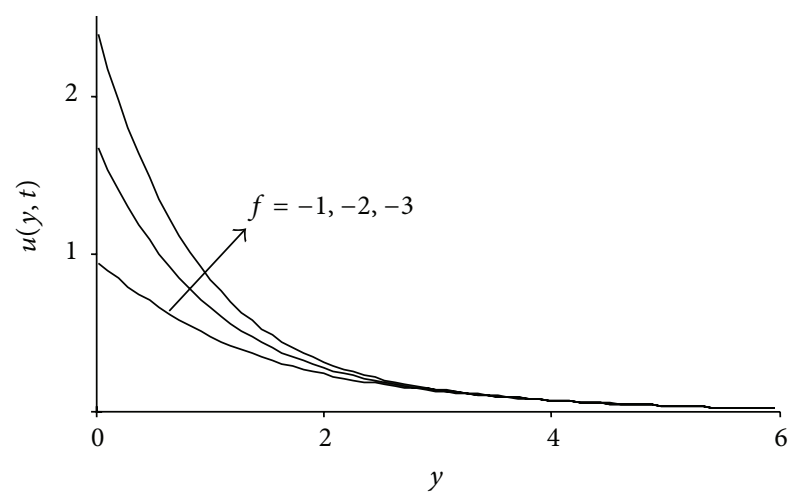

Figure 7: Velocity profiles for $\operatorname{Pr}_{\text {eff }}=0.350\left(N_{r}=1, \operatorname{Pr}=0.7\right)$, $K_{p}=0.7, t=1.2$, and different values of constant shear stress $f$.

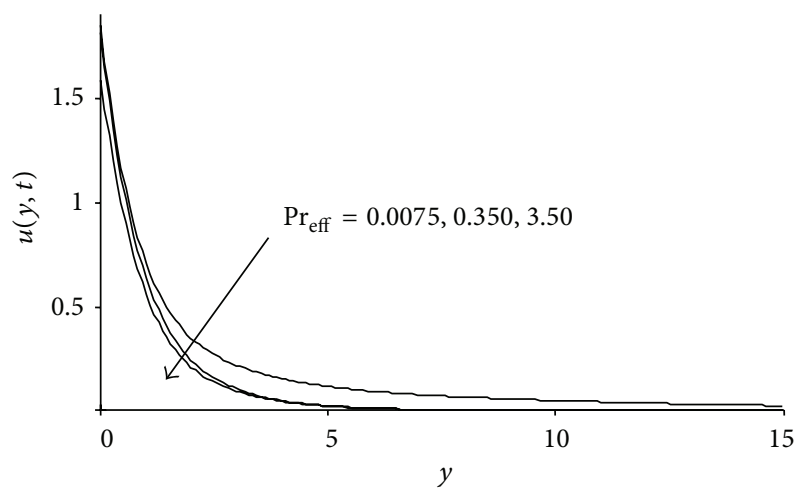

FIGURE 8: Velocity profiles for $K_{p}=0.7, t=0.9$, and different values of $\mathrm{Pr}_{\text {eff }}$ when the plate applies constant shear stress $f=-0.25$.

are plotted. The fluid velocity $u(y, t)$ is written as a sum of two components, that is, convective and mechanical. The velocity solution is further written as a sum of the steady-state and transient solutions $u_{m s}(y, t)$, respectively, $u_{m t}(y, t)$ in the second special case, in which the plate applies an oscillating shear stress to the fluid. Magnetic parameter $M$ slows the fluid flow.

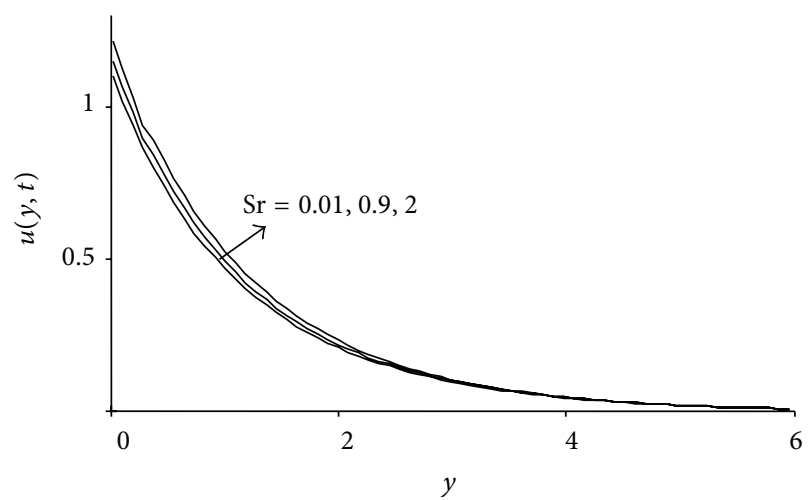

FIGURE 9: Velocity profiles for $K_{p}=0.7, t=0.9$, and different values of $\mathrm{Sr}$ when the plate applies constant shear stress $f=-0.25$.

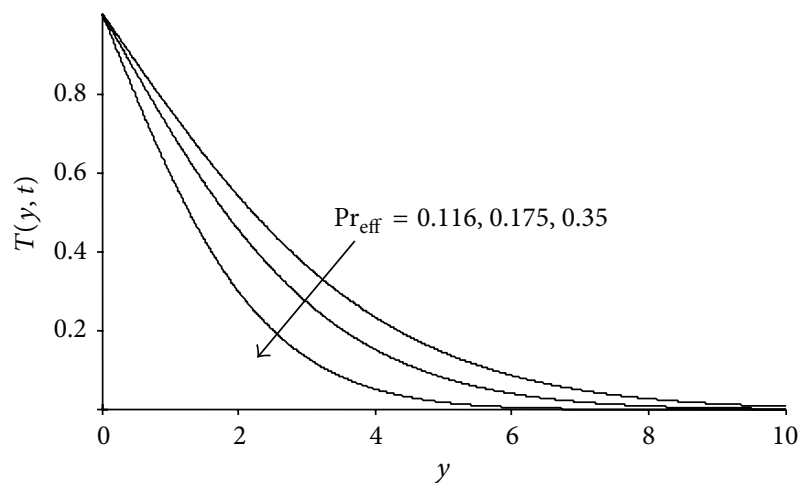

Figure 10: Temperature profile for $t=1.2$ and different values of $\operatorname{Pr}_{\text {eff }}$.

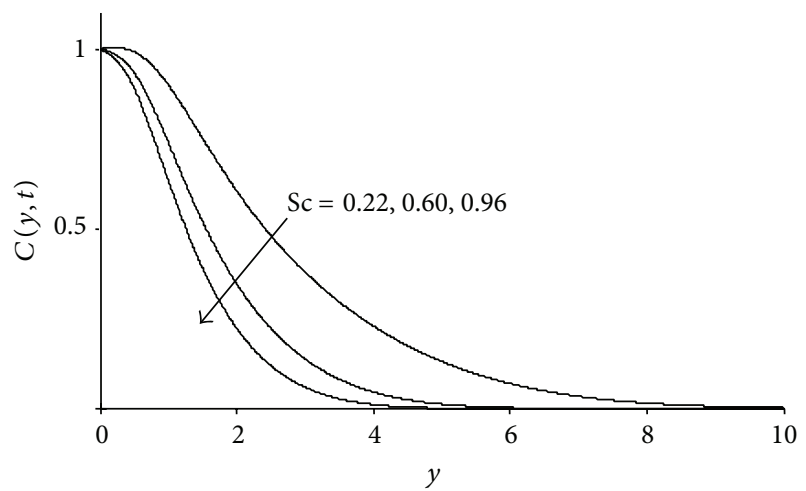

FIGURE 11: Concentration profiles for $t=1.2$ and different values of Sc.

\section{Conflict of Interests}

The authors of this paper do not have any conflict of interests.

\section{Acknowledgments}

The authors would like to acknowledge MOE and Research Management Centre, UTM, for the financial support through Votes nos. $04 \mathrm{H} 27$ and $4 \mathrm{~F} 255$ for this research. 


\section{References}

[1] J. Singh, "Flow of elasto-viscous fluid past an accelerated porous plate," Astrophysics and Space Science, vol. 106, no. 1, pp. 41-45, 1984.

[2] M. Narahari, "Effects of thermal radiation and free convection currents on the unsteady Couette flow between two vertical parallel plates with constant heat flux at one boundary," WSEAS Transactions on Heat and Mass Transfer, vol. 5, no. 1, pp. 21-30, 2010.

[3] M. Narahari and M. Y. Nayan, "Free convection flow past an impulsively started infinite vertical plate with Newtonian heating in the presence of thermal radiation and mass diffusion," Turkish Journal of Engineering and Environmental Sciences, vol. 35, no. 3, pp. 187-198, 2011.

[4] M. Narahari and A. Ishak, "Radiation effects on free convection flow near a moving vertical plate with newtonian heating," Journal of Applied Sciences, vol. 11, no. 7, pp. 1096-1104, 2011.

[5] R. C. Chaudhary and P. Jain, "Unsteady free convection boundary-layer flow past an impulsively started vertical surface with Newtonian heating," Romanian Journal of Physics, vol. 51, p. 911, 2006.

[6] U. N. Das, R. Deka, and V. M. Soundalgekar, "Effects of mass transfer on flow past an impulsively started infinite vertical plate with constant heat flux and chemical reaction," Forschung im Ingenieurwesen, vol. 60, no. 10, pp. 284-287, 1994.

[7] V. M. Soundalgekar, N. S. Birajdar, and V. K. Darwhekar, "Masstransfer effects on the flow past an impulsively started infinite vertical plate with variable temperature or constant heat flux," Astrophysics and Space Science, vol. 100, no. 1-2, pp. 159-164, 1984.

[8] R. Muthucumaraswamy, P. Ganesan, and V. M. Soundalgekar, "On flow and heat transfer of a viscous incompressible fluid past an impulsively started vertical isothermal plate," International Journal of Thermal Sciences, vol. 40, no. 3, pp. 297-302, 2001.

[9] R. Muthucumaraswamy, P. Ganesan, and V. M. Soundalgekar, "Heat and mass transfer effects on flow past an impulsively started vertical plate," Acta Mechanica, vol. 146, no. 1-2, pp. 18, 2001.

[10] S. S. Das, S. R. Biswal, U. K. Tripathy, and P. Das, "Mass transfer effects on unsteady hydromagnetic convective flow past a vertical porous plate in a porous medium with heat source," Journal of Applied Fluid Mechanics, vol. 4, no. 4, pp. 91-100, 2011.

[11] C. J. Toki and J. N. Tokis, "Exact solutions for the unsteady free convection flows on a porous plate with time-dependent heating," Zeitschrift für Angewandte Mathematik und Mechanik, vol. 87, no. 1, pp. 4-13, 2007.

[12] N. Senapati, R. K. Dhal, and T. K. Das, "Effects of chemical reaction on free convection MHD flow through porous medium bounded by vertical surface with slip flow region," American Journal of Computational and Applied Mathematics, vol. 2, pp. 124-135, 2012.

[13] I. Khan, K. Fakhar, and S. Shafie, "Magnetohydrodynamic free convection flow past an oscillating plate embedded in a porous medium," Journal of the Physical Society of Japan, vol. 80, no. 10, Article ID 104401, 2011.

[14] A. Khan, I. Khan, F. Ali, S. Ulhaq, and S. Shafie, "Effects of wall shear stress on unsteady MHD conjugate flow in a porous medium with ramped wall temperature," PLoS ONE, vol. 9, no. 3, Article ID e90280, 2014.
[15] A. Hussanan, Z. Ismail, I. Khan, A. G. Hussein, and S. Shafie, "Unsteady boundary layer MHD free convection flow in a porous medium with constant mass diffusion and Newtonian heating," The European Physical Journal Plus, vol. 129, article 46, 2014.

[16] R. N. Barik, G. C. Dash, and M. Kar, "Free convection heat and mass transfer MHD flow in a vertical porous channel in the presence of chemical reaction," Journal of Fluids, vol. 2013, Article ID 297493, 14 pages, 2013.

[17] P. Chandran, N. C. Sacheti, and A. K. Singh, "Natural convection near a vertical plate with ramped wall temperature," Heat and Mass Transfer, vol. 41, no. 5, pp. 459-464, 2005.

[18] M. Narahari, O. A. Beg, and S. K. Ghosh, "Mathematical modelling of mass transfer and free convection current effects on unsteady viscous flow with ramped wall temperature," World Journal of Mechanics, vol. 1, pp. 176-184, 2011.

[19] V. Rajesh, "Chemical reaction and radiation effects on the transient MHD free convection flow of dissipative fluid past an Infinite vertical porous plate with ramped wall temperature," Chemical Industry \& Chemical Engineering Quarterly, vol. 17, no. 2, pp. 189-198, 2011.

[20] R. R. Patra, S. Das, R. N. Jana, and S. K. Ghosh, “Transient approach to radiative heat transfer free convection flow with ramped wall temperature," Journal of Applied Fluid Mechanics, vol. 5, no. 2, pp. 9-13, 2012.

[21] G. S. Seth, G. K. Mahatoo, and S. Sarkar, "Effects of Hall current and rotation on MHD natural convection flow past an impulsively moving vertical plate with ramped temperature in the presence of thermal diffusion with heat absorption," International Journal of Energy \& Technology, vol. 5, pp. 1-12, 2013.

[22] Samiulhaq, I. Khan, F. Ali, and S. Shafie, "MHD free convection flow in a porous medium with thermal diffusion and ramped wall temperature," Journal of the Physical Society of Japan, vol. 81, no. 4, Article ID 044401, 2012.

[23] N. Ghara, S. Das, S. L. Maji, and R. N. Jana, "Effect of radiation on MHD free convection flow past an impulsively moving vertical plate with ramped wall temperature," American Journal of Scientific and Industrial Research, vol. 3, no. 6, pp. 376-386, 2012.

[24] S. Das, C. Mandal, and R. N. Jana, "Effects of radiation on unsteady Couette flow between two vertical parallel plates with ramped wall temperature," International Journal of Computer Applications, vol. 39, pp. 34-42, 2012.

[25] C. L. M. H. Navier, "Sur les lois dee mouvement des fluids," Mémoires de l'Académie Royale des Sciences de l'Institut de France, vol. 6, pp. 389-440, 1827.

[26] C. Fetecau, C. Fetecau, and M. Rana, "General solutions for the unsteady flow of second-grade fluids over an infinite plate that applies arbitrary shear to the fluid," Zeitschrift für Naturforschung-Section A: Journal of Physical Sciences, vol. 66, no. 12, pp. 753-759, 2011.

[27] T. Hayat, I. Khan, R. Ellahi, and C. Fetecau, "Some MHD flows of a second grade fluid through the porous medium," Journal of Porous Media, vol. 11, no. 4, pp. 389-400, 2008.

[28] E. Magyari and A. Pantokratoras, "Note on the effect of thermal radiation in the linearized Rosseland approximation on the heat transfer characteristics of various boundary layer flows," International Communications in Heat and Mass Transfer, vol. 38 , no. 5, pp. 554-556, 2011. 
[29] A. David Maxim Gururaj and S. P. Anjali Devi, "MHD boundary layer flow with forced convection past a nonlinearly stretching surface with variable temperature and nonlinear radiation effects," International Journal of Development Research, vol. 4, pp. 75-80, 2014.

[30] C. Fetecau, M. Rana, and C. Fetecau, "Radiative and porous effects on free convection flow near a vertical plate that applies shear stress to the fluid," Zeitschrift für Naturforschung-Section A: Journal of Physical Sciences, vol. 68, no. 1-2, pp. 130-138, 2013. 


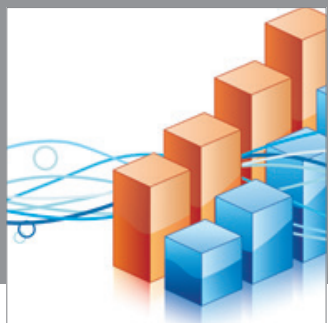

Advances in

Operations Research

mansans

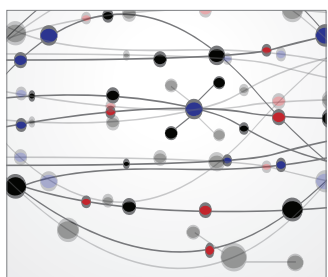

The Scientific World Journal
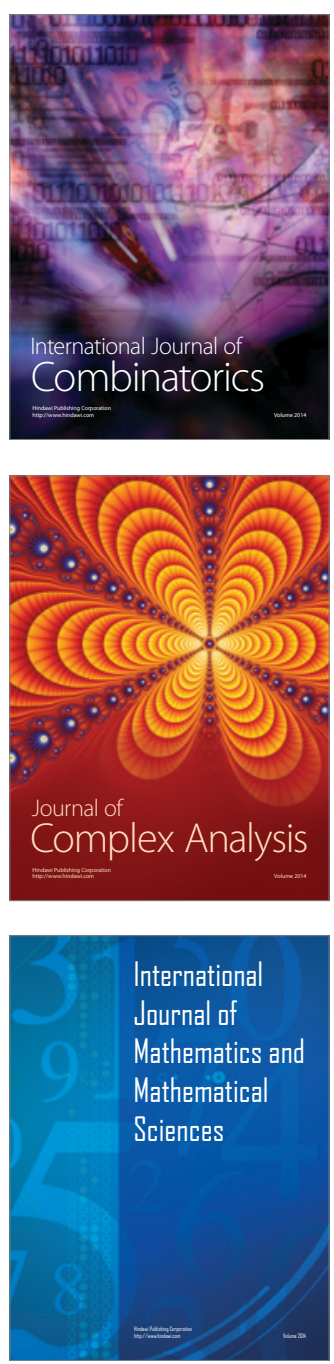
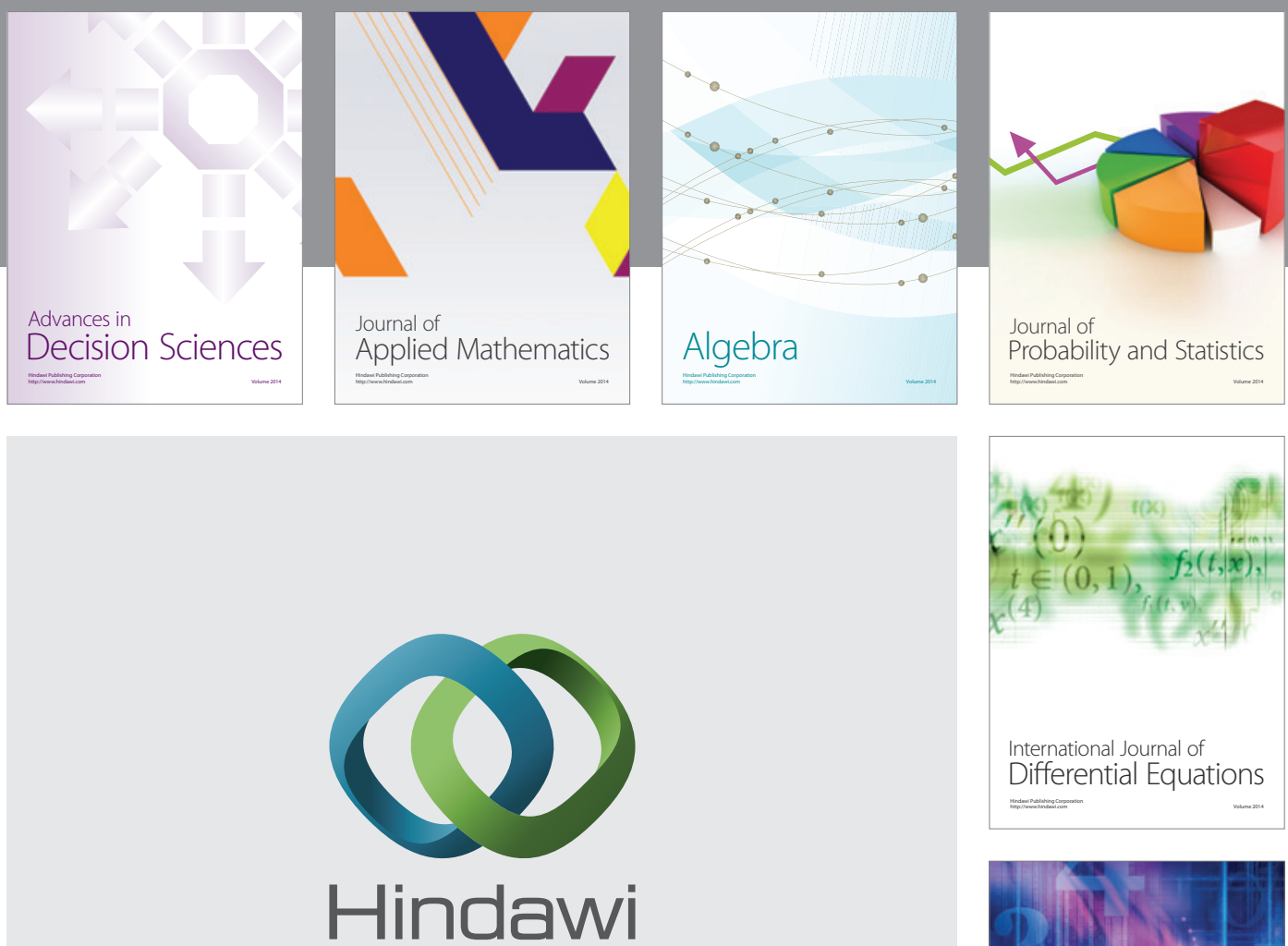

Submit your manuscripts at http://www.hindawi.com
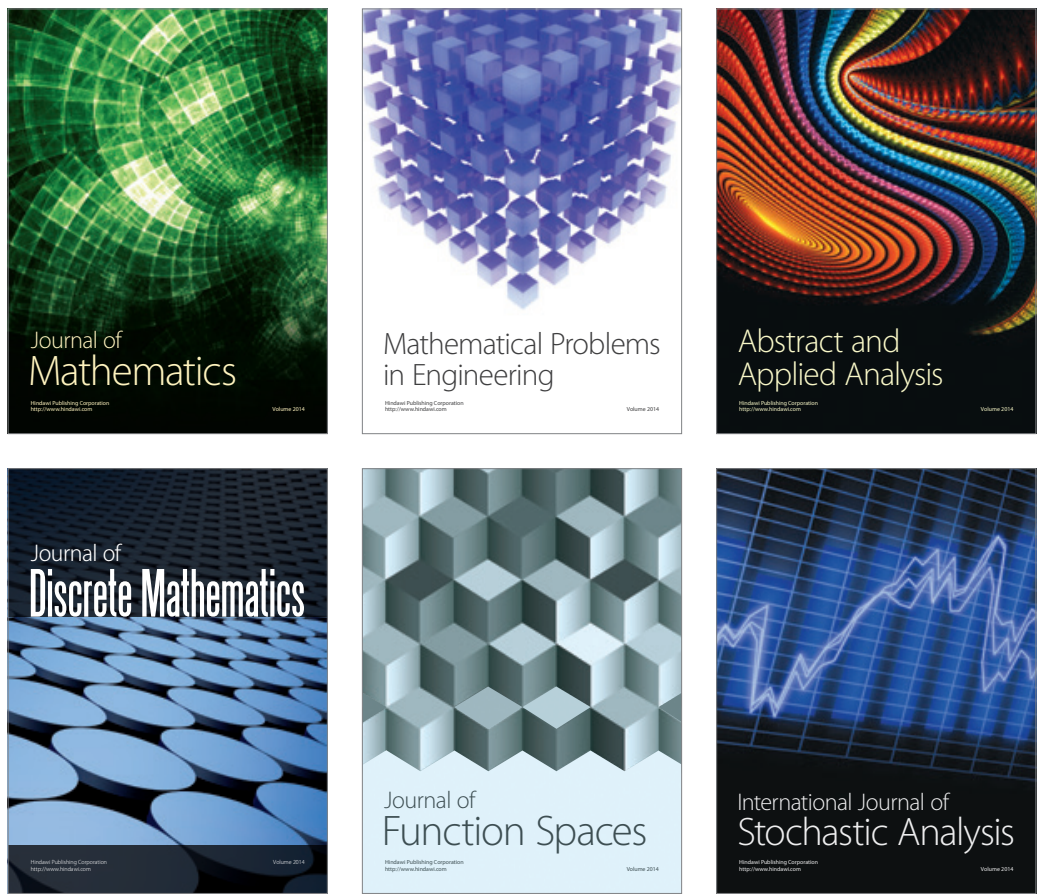

Journal of

Function Spaces

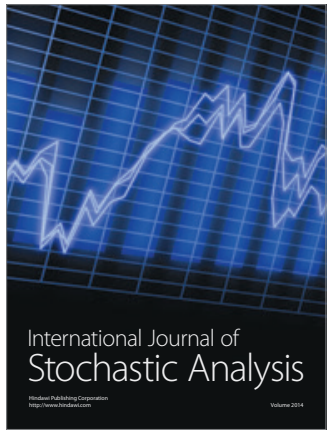

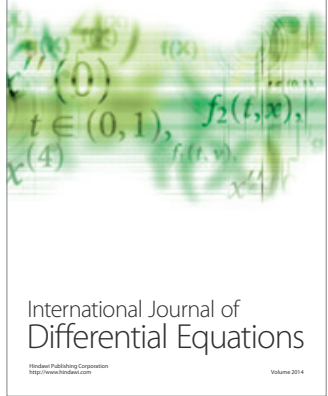
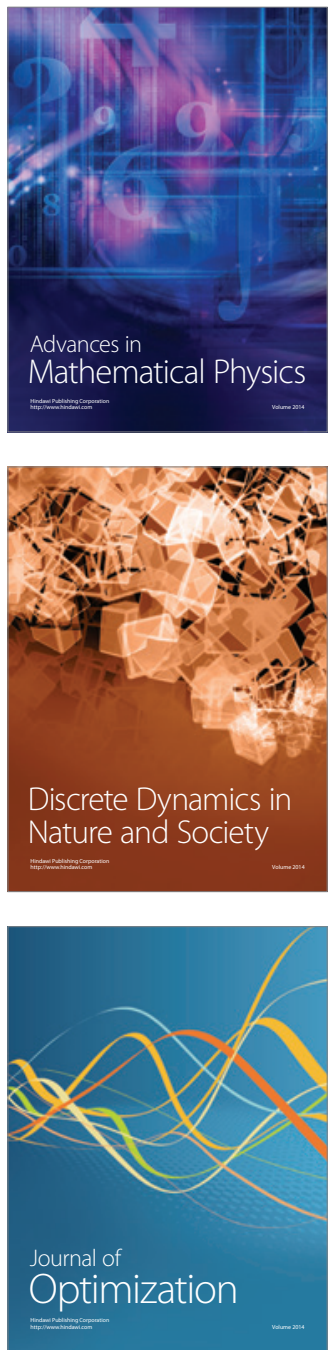